\title{
Flow Modulation and Mixing Enhancement of Highly Underexpanded Jet by Vortex Excitation
}

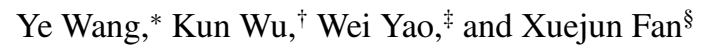 \\ State Key Laboratory of High Temperature Gas Dynamics, Institute of Mechanics, Chinese \\ Academy of Sciences, 100190 Beijing, People's Republic of China
}

https://doi.org/10.2514/1.J058476

\begin{abstract}
A highly underexpanded jet with a nozzle pressure ratio of 5.60 is excited by a vortex excitation. Modulations on flow characteristics by the vortex excitation are revealed by comparing the excited jet with the free jet through a large-eddy simulation (LES) technique. The modeling results have achieved good agreements with the available literature data in terms of time-averaged near-field variables, Mach disk dimensions, and dominant frequencies. A grid-convergence study is also performed to verify the fidelity of LES results. Ultimately, qualitative and quantitative comparisons of mean and instantaneous jet fields are conducted. It is demonstrated that the vortex excitation has enhanced the mixing of the injection nitrogen with the environment by a considerably increased mixing level and a faster mixing process in the middle jet. The vortex excitation modulates the buildup process of shocks and vortex structures, resulting in an intensified shock/shear-layer interaction and an accelerated turbulence transition process, which combine to improve mixing efficiency. The dominant instability mode is altered to a higher-order multiple helical mode from a single helical mode, and an intensified screech tone is achieved by the vortex excitation.
\end{abstract}

\section{Nomenclature}

$\begin{array}{ll}A & =\text { mixing area, } \mathrm{m}^{2} \\ C_{\varepsilon}, C_{v} & =\text { model constants, dimensionless } \\ D & =\text { nozzle exit diameter, } \mathrm{m} \\ D_{k m} & =\text { mass diffusion coefficient between species } k \text { and } m, \\ & \\ D_{m} \cdot \mathrm{s}^{-1} \\ E_{t} & =\text { diameter of Mach disk, } \mathrm{m} \\ f & =\text { total energy, } \mathrm{m}^{2} \cdot \mathrm{s}^{-2} \\ H & =\text { frequency, } \mathrm{Hz} \\ H_{i} & =\text { helicity, } \mathrm{m} \cdot \mathrm{s}^{-2} \\ H_{m} & =\text { height of Mach in disk, } \mathrm{m} \\ h_{t} & =\text { total enthalpy, } \mathrm{m}^{2} \cdot \mathrm{s}^{-2} \\ k & =\text { turbulent kinetic energy, } \mathrm{m}^{2} \cdot \mathrm{s}^{-3} \\ M a & =\text { Mach number, dimensionless } \\ P r & =\text { Prandtl number, dimensionless } \\ P r_{t} & =\text { turbulent Prandtl number, dimensionless } \\ p & =\text { pressure, Pa } \\ Q & =Q \text { criterion, } \mathrm{s}^{-2} \\ q_{i} & =\text { heat flux in } x_{i}, \mathrm{~kg} \cdot \mathrm{s}^{-3} \\ R & =\text { gas constant of the mixture, } \mathrm{m}^{2} \cdot \mathrm{s}^{-2} \cdot \mathrm{K}^{-1} \\ R e & =\text { Reynolds number, dimensionless } \\ S c_{k} & =\text { Schmidt number of species } k, \text { dimensionless } \\ S c_{k, t} & =\text { turbulent Schmidt number of species } k, \text { dimensionless } \\ T & =\text { temperature, } \mathrm{K} \\ t_{0} & =\text { integral time scale, } \mathrm{s}\end{array}$

Received 23 March 2019; revision received 27 December 2019; accepted for publication 24 February 2020; published online 13 May 2020. Copyright (C) 2020 by the American Institute of Aeronautics and Astronautics, Inc. All rights reserved. All requests for copying and permission to reprint should be submitted to CCC at www.copyright.com; employ the eISSN 1533-385X to initiate your request. See also AIAA Rights and Permissions www.aiaa.org/ randp.

*Graduate Student; also School of Engineering Science, University of the Chinese Academy of Sciences, 100049 Beijing, People's Republic of China; wangye@imech.ac.cn.

Assistant Professor; also School of Engineering Science, University of the Chinese Academy of Sciences, 100049 Beijing, People's Republic of China; wukun@imech.ac.cn.

${ }^{\ddagger}$ Associate Professor; also School of Engineering Science, University of the Chinese Academy of Sciences, 100049 Beijing, People's Republic of China; weiyao@imech.ac.cn.

\$Professor; also School of Engineering Science, University of the Chinese Academy of Sciences, 100049 Beijing, People's Republic of China; xfan@ imech.ac.cn (Corresponding Author).

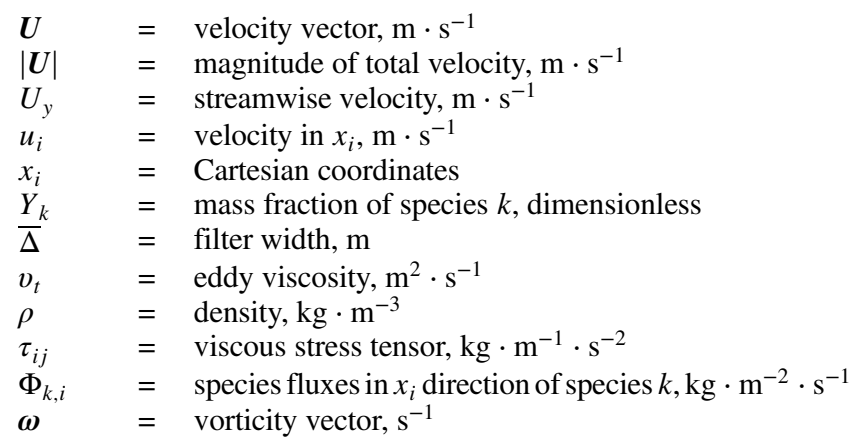

Subscripts

$\begin{array}{lll}e & = & \text { condition at nozzle exit } \\ 0 & = & \text { stagnation condition } \\ \infty & = & \text { ambient condition }\end{array}$

Superscript

sgs $=$ subgrid-scale quantities

\section{Introduction}

$\mathbf{T}$ HE highly underexpanded jet [1] is frequently encountered in high-speed propulsion systems, such as the fuel/air mixing in scramjets $[2,3]$. Driven by a nozzle pressure ratio beyond 3.85 [4], highly underexpanded jets show complicated flow characteristics including near-field shocks and unsteady coherent structures in shear layer, which plays a vital role in the mixing process of a fuel jet with air. For a scramjet, an ultrafast and sufficient fuel/air mixing in highly underexpanded jets is particularly required to achieve autoignition and increase combustion efficiency $[2,5,6]$. Thus, focusing on this issue, we previously conducted research on the unsteady time evolution and instability characteristics of free highly underexpanded jets [7]. The findings help to understand the detailed jet development mechanism, and are thus of great benefit for mixing enhancement designs [2]. Accordingly, a mixing enhancement method by sinusoidal pressure disturbances at jet inherent frequencies (e.g., the shock screech frequencies) was imposed to excite a highly underexpanded jet [8]. However, the mixing performance was just marginally improved by the pressure disturbances, although remarkable changes on some flow characteristics occurred. Therefore, as a continuation, the aim of the present research is to explore a 
new and effective approach to the fuel/air mixing problem in highly underexpanded jets.

A large body of work has been devoted to the mixing problem for decades [2,9]. It has been recognized that mixing enhancement can be achieved by jet excitation, which introduces external disturbances to the jet flow to excite the shear-layer instability and promote the jet/air interaction, and thus enhance mixing [10-12]. In addition to the aforementioned excitation introducing acoustic disturbances [8], there are two other excitation methods according to the decomposition of disturbances [13], i.e., entropy and vortex excitations that introduce entropy and vorticity disturbances, respectively.

The acoustic excitation is the most frequently reported; apart from the previous study by our group on the highly underexpanded jet [8], Murugappan et al. [14] used a high-frequency actuator to impose acoustic excitations to a supersonic transverse jet flow at Mach 2.0, where periodic eddies induced in the shear layer provided superior mixing performance and higher penetration depth. The entropy excitation was investigated by Mingbo et al. [15] through enforcing periodic temperature perturbations on a two-dimensional supersonic mixing layer. When the convective Mach number was less than one, the mixing effect was enhanced with the growth of spanwise vortices. Regarding the third one, most of the passive control techniques are based on the vortex excitation, which induces vortices and augments vorticity disturbances to excite the flow, e.g., those strut-based [16,17], cavity-based [18], and ramp-based [19-22] injections, where streamwise vortices are induced to excite the large-scale entrainment between different streams, and therefore enhance mixing. The detailed mechanism of those strategies was reviewed by Huang [23]. Considering our unsatisfactory trial on the acoustic one and the uncertainty on high-speed mixing of the entropy one, the vortexexcitation mechanism (easier to achieve efficient mixing) is preferred in the present study.

Besides the streamwise vortices, hoop vortices can promote mixing through intensifying Kelvin-Helmholtz (K-H) instability in the shear layer, and therefore a greater mixing can be obtained if there is an excitation simultaneously augmenting both types of vortices in jet flow. Inspired by the experiments of Strykowski and Niccum [24], Strykowski and Wilcoxon [25], and Strykowski et al. [26], we noticed that the counterflow excitation method meets the aforementioned conditions with the aid of a hoop vortex generated near the nozzle. Strykowski and Wilcoxon [25] and Strykowski et al. [26] demonstrated the feasibility and efficiency of the counterflow excitation by showing a large improvement of mixing in subsonic jets and slightly underexpanded supersonic jets. To interpret the mixing enhancement mechanism, they focused on stability analysis but not the mechanism of the hoop vortex-based excitation [24]. However, concerning the highly underexpanded jet studied here, vortices significantly modulate its flow characteristics, and thus the mixing performance owing to its high-speed and high-turbulence features $[4,27]$. Therefore, the hoop vortex-based physics needs to be uncovered here.

In this study, a counterflow is employed to impose the hoop vortexbased excitation to a highly underexpanded jet. Compressible largeeddy simulation (LES) modelings are conducted on the baseline jet and the vortex-excited jet. The time-averaged flowfields are compared first, and then three aspects of modulations by the vortex excitation on the highly underexpanded jet are concentrated on: 1) the mixing performance; 2) the transitional features including near-nozzle waves and vortex structures; and 3) the dominant instability characteristics. In addition, the baseline jet is consistent with previous studies by our group $[\underline{7}, \underline{8}]$, and some results are compared with them.

\section{Computational Approach}

\section{A. Numerical Methodology}

The large-eddy simulation was employed to model the unsteady evolution of the compressible and multicomponent flow, which is governed by the three-dimensional filtered Navier-Stokes equations describing the conservation of mass, momentum, energy, and species, respectively, listed in Eqs. (1-4). The ideal gas state equation [Eq. (5)] is used to relate the filtered pressure, temperature, and density, with the subgrid-scale (SGS) density term neglected because the flow considered is far from the drastic phase-change region:

$$
\begin{gathered}
\frac{\partial \bar{\rho}}{\partial t}+\frac{\partial \bar{\rho} \tilde{u}_{i}}{\partial x_{i}}=0 \\
\frac{\partial \bar{\rho} \tilde{u}_{i}}{\partial t}+\frac{\partial \bar{\rho} \tilde{u}_{i} \tilde{u}_{j}}{\partial x_{j}}=-\frac{\partial \bar{p}}{\partial x_{i}}+\frac{\partial \bar{\tau}_{i j}}{\partial x_{j}}-\frac{\partial \tau_{i j}^{\mathrm{sgs}}}{\partial x_{j}} \\
\frac{\partial \bar{\rho} \tilde{h}_{t}}{\partial t}+\frac{\partial \bar{\rho} \tilde{u}_{j} \tilde{h}_{t}}{\partial x_{j}}=\frac{\partial \bar{p}}{\partial t}+\frac{\partial \tilde{u}_{j} \tilde{\tau}_{i j}}{\partial x_{i}}-\frac{\partial \bar{q}_{i}}{\partial x_{i}}-\frac{\partial H_{i}^{\mathrm{sgs}}}{\partial x_{i}} \\
\frac{\partial \bar{\rho} \tilde{Y}_{k}}{\partial t}+\frac{\partial \bar{\rho} \tilde{u}_{j} \tilde{Y}_{k}}{\partial x_{j}}=\frac{\partial}{\partial x_{j}}\left(\bar{\rho} D_{k m} \frac{\partial \tilde{Y}_{k}}{\partial x_{j}}\right)-\frac{\partial \Phi_{k, j}^{\mathrm{sgs}}}{\partial x_{j}} \\
\bar{p}=\bar{\rho} R \tilde{T}
\end{gathered}
$$

where the overbar "-" denotes spatially filtered variables, whereas the tilde " $\sim$ " denotes Favre-filtered variables. Here, $\rho$ represents the density, $p$ the pressure, $u_{i}$ the velocity in the $x_{i}$ direction, and $\tau_{i j}$ the viscous stress tensor. In the energy equation, $h_{t}=h+u_{i} u_{i} / 2=E_{t}+p / \rho$ is the total enthalpy, $h$ is the static enthalpy, $E_{t}$ is the total energy, $q_{i}$ is the heat flux, and $R$ is the gas constant of the mixture. In the mass transport equation, $Y_{k}$ and $D_{k m}$ denote the mass fraction of species $k$ and the mass diffusion coefficient between species $k$ and $m$.

The thermodynamic properties, such as the enthalpy and the specific heat of species, are obtained from the NIST-JANAF thermophysical table [28]. The viscosity of the species is computed using Sutherland's law, and the thermal and mass diffusivities are estimated by assuming a constant Prandtl number $(\operatorname{Pr}=0.7)$ and Schmidt number $\left(S c_{k}=0.7\right)$.

All terms with the superscript "sgs" indicate the SGS quantities of turbulence that require additional modeling. The SGS energy fluxes $H_{i}^{\mathrm{sgs}}=\left(\bar{\rho} \tilde{E}_{t} \tilde{u}_{i}-\bar{\rho} \tilde{E}_{t} \tilde{u}_{i}\right)+\left(\rho \bar{u}_{i}-\bar{\rho} \tilde{u_{i}}\right)$ and the SGS species fluxes $\Phi_{k, j}^{\text {sgs }}=\bar{\rho} u_{j} \tilde{Y}_{k}-\bar{\rho} \tilde{u}_{j} \tilde{Y}_{k}$, are modeled by the standard gradient diffusion assumption:

$$
\begin{gathered}
H_{j}^{\mathrm{sgs}}=-\bar{\rho} \frac{\nu_{t}}{P r_{t}} \frac{\partial \tilde{H}}{\partial x_{j}}=-\bar{\rho} \frac{\nu_{t}}{P r_{t}}\left(\frac{\partial \tilde{h}}{\partial x_{j}}+\tilde{u}_{i} \frac{\partial \tilde{u}_{i}}{\partial x_{j}}+\frac{\partial k^{\mathrm{sgs}}}{\partial x_{j}}\right) \\
\Phi_{k, j}^{\mathrm{sgs}}=-\bar{\rho} \frac{\nu_{t}}{S c_{k, t}} \frac{\partial \tilde{Y}_{k}}{\partial x_{j}}
\end{gathered}
$$

where $\nu_{t}$ is the eddy viscosity. The turbulent Prandtl number $\operatorname{Pr}_{t}$ and turbulent Schmidt number $S c_{k, t}$ are both set to 0.7. Another SGS term pertaining to the momentum equation is the SGS stress term $\tau_{i j}^{\mathrm{sgs}}=\bar{\rho}\left(\widetilde{u_{i} u_{j}}-\tilde{u}_{i} \tilde{u}_{j}\right)$, which is modeled by the Boussinesq eddy viscosity hypothesis:

$$
\tau_{i j}^{\mathrm{sgs}}=-2 \nu_{t} \bar{\rho}\left[\tilde{S}_{i j}-\frac{\delta_{i j}}{3} \tilde{S}_{k k}\right]+\frac{2 \delta_{i j}}{3} \bar{\rho} k^{\mathrm{sgs}}
$$

where $k^{\mathrm{sgs}}$ is the SGS turbulent kinetic energy, and $\tilde{S}_{i j}$ is the strain tensor for the resolved scale:

$$
\tilde{S}_{i j}=\frac{1}{2}\left(\frac{\partial \tilde{u}_{i}}{\partial x_{j}}+\frac{\partial \tilde{u}_{j}}{\partial x_{i}}\right)
$$

To better capture the nonequilibrium kinetic energy transfer in the high-speed shear layer of the highly underexpanded jets, the one-equation turbulent kinetic energy model $[29,30]$ governed by Eqs. (10) and (11) is employed to close the SGS stress term: 


$$
\frac{\partial \bar{\rho} k^{\mathrm{sgs}}}{\partial t}+\frac{\partial \bar{\rho} \tilde{u}_{j} k^{\mathrm{sgs}}}{\partial x_{j}}=\frac{\partial}{\partial x_{j}}\left[\bar{\rho}\left(\frac{\nu_{t}}{P r_{t}}+\nu\right) \frac{\partial k^{\mathrm{sgs}}}{\partial x_{j}}\right]-\tau_{i j}^{\mathrm{sgs}} \frac{\partial \tilde{u}_{i}}{\partial x_{j}}-C_{\varepsilon} \frac{\bar{\rho}\left(k^{\mathrm{sgs}}\right)^{3 / 2}}{\bar{\Delta}}
$$

$$
\nu_{t}=C_{\nu} \bar{\Delta} \sqrt{k^{\mathrm{sgs}}}
$$

where $C_{\varepsilon}=1.048$ and $C_{\nu}=0.094$ are model constants, and $\bar{\Delta}$ is the filter width. Comparison of the different SGS models by Fureby et al. [31] shows that the one-equation model performs better than zero-equation models in modeling transition and unsteadiness in turbulence. Two-equation models (e.g., the shear-stress transport $k-\omega$ model) do well in predicting supersonic transverse jet flows where the wall effect should be taken into significant consideration $[32,33]$. In the present study, we do not focus on the wall effect of the nozzle; thus, we choose the one-equation turbulent kinetic energy model for its efficiency.

The governing equations are solved by the in-house-developed finite volume compressible LES solver AstroFoam, which is extended from the standard compressible solver rhoCentralFoam distributed with the open-source computational fluid dynamics package OpenFOAM V3.0.1 [34], mainly by adding the modules of species transportation and chemical reaction with realistic thermodynamics and transport properties. The nonlinear inviscid convective fluxes are evaluated by using a second-order semidiscrete central KurganovTadmor scheme [35]. A second-order spatial accuracy in reconstructing primitive convective fluxes at faces is achieved by the total variation diminishing scheme. The temporal integration is advanced by the second-order Crank-Nicholson scheme [36]. The accuracy of AstroFoam has been extensively validated for various supersonic flows $[\underline{7}, \underline{8}, \underline{37}-\underline{39}]$ and supersonic combustions $[\underline{40}, \underline{41}]$.

\section{B. Numerical Setup}

Figure 1a depicts the computational domain in a cuboid shape with the dimensions of 50,100, and $50 \mathrm{~mm}$ in the $x, y$, and $z$ directions, respectively. The coordinate $y$ denotes the streamwise direction, whereas $x$ and $z$ denote the spanwise directions. The center of the jet orifice is located at the origin $y=0$ and the radial coordinate $r=0$. To be consistent with our previous studies $[\underline{7}, \underline{8}]$, the primary jet of pure nitrogen $\left(\mathrm{N}_{2}\right)$ with a total pressure of $p_{0}=5.6$ bar and a total temperature of $T_{0}=360 \mathrm{~K}$ is issued from a convergent nozzle, which has a height of $20 \mathrm{~mm}$ and an exit diameter of $D=2 \mathrm{~mm}$.
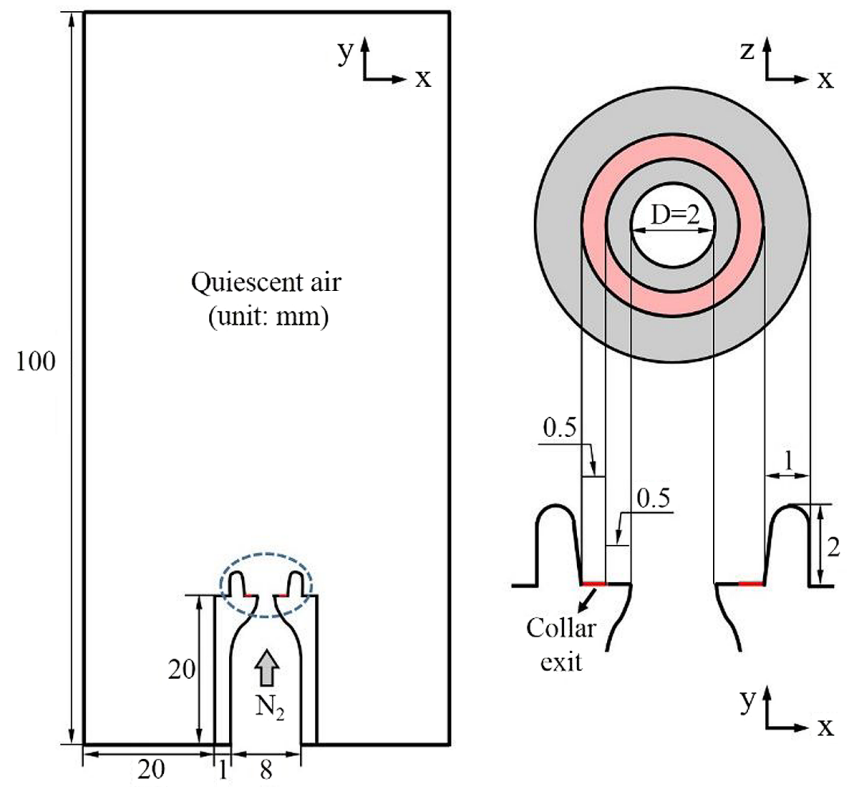

a)

b)

Fig. 1 Schematic diagram of a) computational domain and b) the enlarged nozzle-collar part.
The domain is initially filled with quiescent air in the mass composition of $76.699 \%$ nitrogen and $23.301 \%$ oxygen, as well as at the pressure of $p_{\infty}=1 \mathrm{bar}$, the temperature of $T_{\infty}=300 \mathrm{~K}$, and the density of $\rho_{\infty}=1.17 \mathrm{~kg} / \mathrm{m}^{3}$.

The enlarged part of the nozzle exit in Fig. $1 \mathrm{~b}$ is the nozzle-collar system, which stems from the experiment of Strykowski and Wilcoxon [25] and is optimized here to introduce the vortex excitation. To be specific, as schematically shown in Fig. 2, the vortex excitation is imposed through the near-nozzle hoop vortex (denoted by the blue curves), which is generated by the shear stress between the primary flow and the counterflow in the annular flow passage. Acting as the vortex-excitation source, the hoop vortex evolves into streamwise vortices due to stretching force from streamwise momentum; it also expands and propagates downstream under shear-layer expansion and convective transport. Consequently, additional streamwise and circumferential vorticity disturbances are simultaneously introduced to excite the flow. The counterflow in the nozzle-collar system is driven by a negative pressure difference on either side of the collar exit, which is denoted by red in Figs. 1 and 2 , and has a width of $0.5 \mathrm{~mm}$ bounded from the primary jet $\bar{b}$ y a $0 . \overline{5}-\mathrm{mm}$-wide annular wall. The outer wall of the suction collar has a width of $1 \mathrm{~mm}$ and a protrusion of $2 \mathrm{~mm}$ to confine the counterflow in the periphery of the primary jet, which facilitates the formation of the hoop vortex. In modeling, the jet flow is regarded as an excited jet or free jet, depending on whether the vortex excitation has been activated. For the excited jet, a suction pressure (which is $p_{s}=0.5$ bar in this study) lower than the inside pressure is specified on the collar exit. For the free jet, the collar exit is treated as the wall condition. Note that we hold a constant suction pressure that generates an unsteady counterflow as the inside pressure is coupled with the jet's main flow. This is different from the counterflow with a constant flow rate used by Strykowski and Niccum [24] and Strykowski and Wilcoxon [25].

As shown in Fig. 3 , the unstructured mesh consisting of hexahedral cells is clustered around the injection orifice, the jet core, and the shear layer. The refinement region starts from $y / D=-1$ to 7.5 in the streamwise direction, and from $r / D=0$ to 2 in the radial direction. The cell size is gradually stretched toward the lateral boundaries and the outlet in order to provide appropriate numerical dissipation to damp out the wave reflections from these boundaries. The minimal and maximal cell sizes are 0.03 and $0.1 \mathrm{~mm}$ in the streamwise direction, and they are 0.02 and $0.08 \mathrm{~mm}$ in the radial direction. Three mesh sets with 10,20, and 30 million cells are employed for the grid-convergence study.

Details of the boundary conditions are summarized in Table 1 . The current stagnation pressure of the primary jet results in a nozzle pressure ratio (NPR $=p_{0} / p_{\infty}$ ) of 5.60, which is classified as the highly underexpanded jet (NPR > 3.57) [42] and features a typical

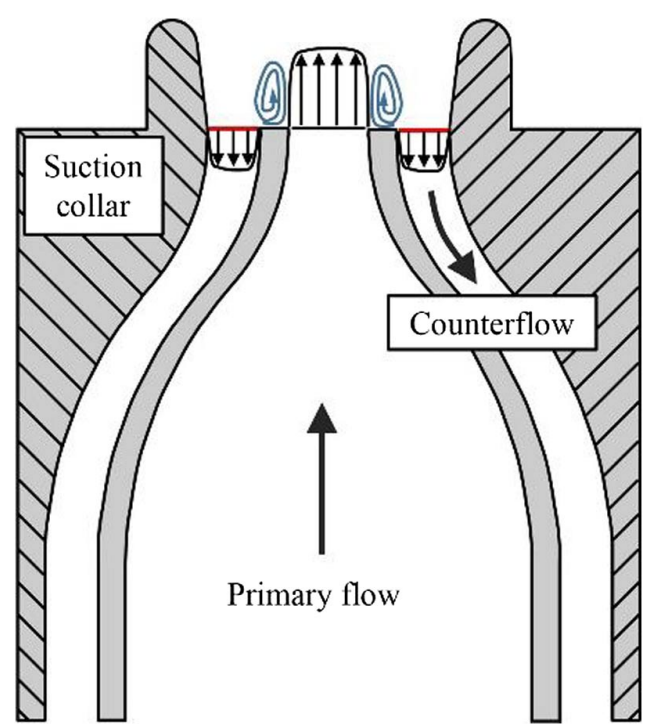

Fig. 2 Schematic diagram of nozzle-collar system. 


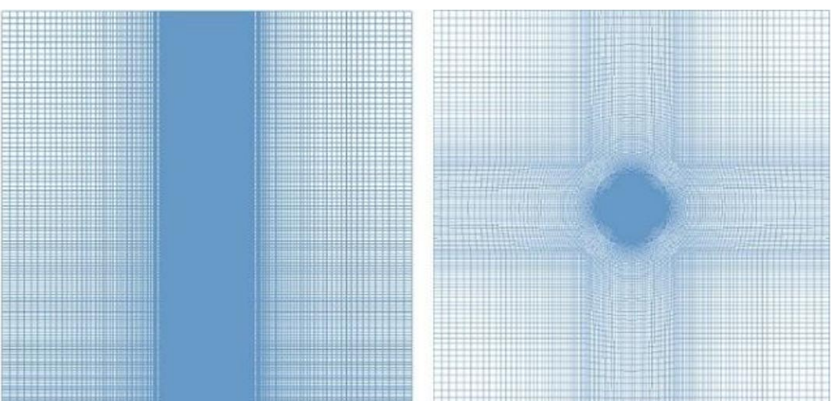

b)

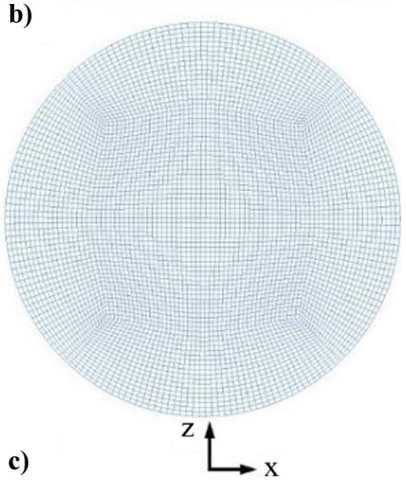

Fig. 3 Unstructured mesh on a) the streamwise plane, b) the bottom plane, and c) the jet orifice.

Table 1 Flow parameters in simulations

\begin{tabular}{lcc}
\hline \hline Parameters & Free jet case & Excited jet case \\
\hline Nozzle pressure ratio; $p_{0} / p_{\infty}$ & 5.6 & 5.6 \\
Suction pressure ratio; $p_{s} / p_{\infty}$ & -- & 0.5 \\
Nozzle temperature ratio; $T_{0} / T_{\infty}$ & 1.2 & 1.2 \\
Streamwise velocity at nozzle exit $U y_{e} \mathrm{~m} / \mathrm{s}$ & 346 & 346 \\
Mach number at nozzle exit $M a_{e}$ & 1.0 & 1.0 \\
Reynolds number at nozzle exit $R e_{e}\left(\times 10^{5}\right)$ & 1.36 & 1.36 \\
\hline \hline
\end{tabular}

single helical mode [7]. In the modeling, stagnation conditions of pressure and temperature are specified on the inlet of the primary jet, which will transit into a sonic flow at the throat, i.e., the inner nozzle exit. In the case of the excitation activated, the pressure on the collar exit is held constant, whereas zero-gradient boundary conditions are specified for the other flow variables. For the free jet case, the collar exit is treated as an adiabatic nonslip wall, as well as all the shells of the nozzle-collar system. The open boundary condition is applied to the lateral side, the upstream free boundary, and the outlet, where the zero-gradient condition is used for the outflow and the ambient air conditions are specified when backflow occurs.

The parallel simulations are performed using $224 \mathrm{CPU}$ cores of Tianhe-1 supercomputer (TH-1) in the National Supercomputer Center of Tianjin. The integration is advanced with a maximum Courant number of 0.3 , corresponding to an average time step of approximately $7.0 \times 10^{-9} \mathrm{~s}$. Each modeling case contains four flowthrough times (FTTs), which cost about 114 CPU months, to obtain stationary statistics. One FTT that the jet flushes out of the entire domain from the inlet is about $0.5 \mathrm{~ms}\left(200 t_{0}\right)$ for the free jet and $0.8 \mathrm{~ms}\left(320 t_{0}\right)$ for the excited jet. The integral time scale $t_{0}$ representing the mean size of the energy-containing anisotropic large eddies is defined as $t_{0}=D / 2\left|\boldsymbol{U}_{e}\right| \approx 2.5 \times 10^{-6} \mathrm{~s}$, where $\left|\boldsymbol{U}_{e}\right|$ denotes the magnitude of total velocity at the nozzle exit. The near-field structure reaches the quasi-steady state after $150 t_{0}$ for the free jet and $200 t_{0}$ for the excited jet.

\section{Numerical Validation}

To verify the numerical setups, an initial numerical validation was conducted for a similar highly underexpanded jet with NPR $=5.74$ against the experiment by Panda and Seasholtz [43]. As compared in Fig. 4, the axial and radial profiles of the time-averaged density are all in good agreement with the experimental data, albeit with a slight underprediction of the intensities of the second and third shocks. The radial profiles indicate that the width and the height of Mach disk, as well as the intensities of near-nozzle structures, have been well captured.

\section{Results and Discussion}

\section{A. Grid-Convergence Study}

Because the excited jet suffers more from the numerical instability compared with the free jet, the grid-convergence study was based on the results from excited jet simulation. Figures $\underline{5}$ and $\underline{6}$ present the time-averaged results predicted by the three different mesh sets, including streamwise velocity $\bar{U}_{y}$, pressure $\bar{p}$, temperature $\bar{T}$, and subgrid-scale turbulent kinetic energy $\bar{k}^{\text {sgs }}$. These results are along spanwise and streamwise axes, and the spanwise axes are at $y / D=1,4$, and 10 , which correspond to regions close to the nozzle exit, after the first shock, and at the middle jet, respectively. In the following analysis, the temperature and pressure are normalized by the initial ambient values, whereas the streamwise velocity is scaled by the value at the jet orifice. The same normalization method will be abided by in the subsequent sections. In Figs. 5 and 6 a, the coarse mesh with 10 million cells shows discernable discrepancies, whereas the medium mesh with 20 million cells well reproduces the results from the finest mesh for streamwise velocity, pressure, temperature, and SGS kinetic energy. Figure 6a also shows that the SGS turbulent kinetic energy varies dramatically near the wave structures and peaks at the first Mach disk, implying that a proper mesh refinement near the Mach disk should be configured to better capture the turbulent kinetic energy cascade. Figure $6 \mathrm{~b}$ reveals that all the mesh sets well predict the cascade of turbulent kinetic energy in accordance with the $-5 / 3$ power law, and a little more energy dissipation is found in the coarsest mesh. To sum up, the negligible differences between the medium and the finest meshes fully demonstrate that their spatial resolutions are adequate to capture the sharp discontinuity and turbulence structures in supersonic jet flow. As a compromise between
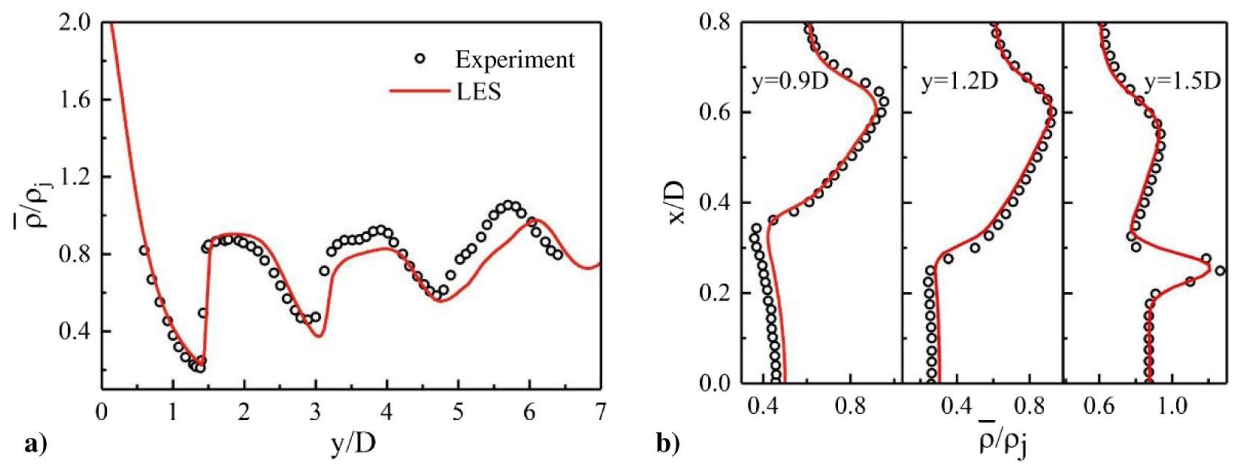

Fig. 4 Profiles of time-averaged density normalized by the fully expanded density $\rho_{j}:$ a) axial and b) radial. 


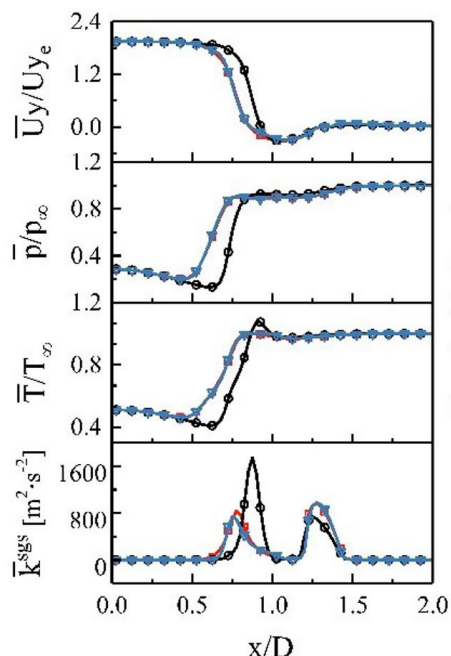

a) $y / D=1$

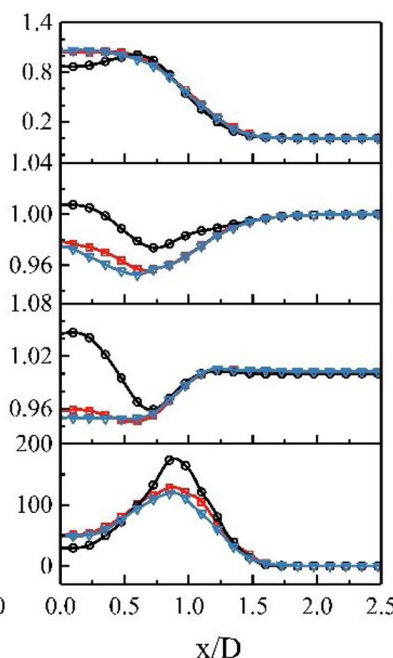

b) $y / D=2$

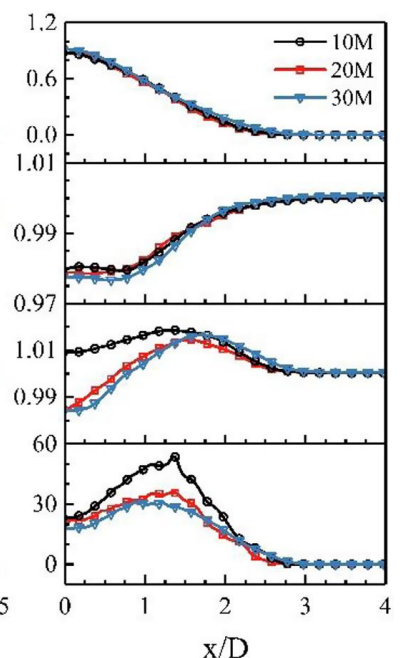

c) $y / D=10$

Fig. 5 Time-averaged spanwise flow variables for the excited jet at $y / D=1,4$, and 10 for the three mesh resolutions. In the legend, $10 \mathrm{M}, 20 \mathrm{M}$, and $30 \mathrm{M}$ mean 10, 20, and 30 million cells.
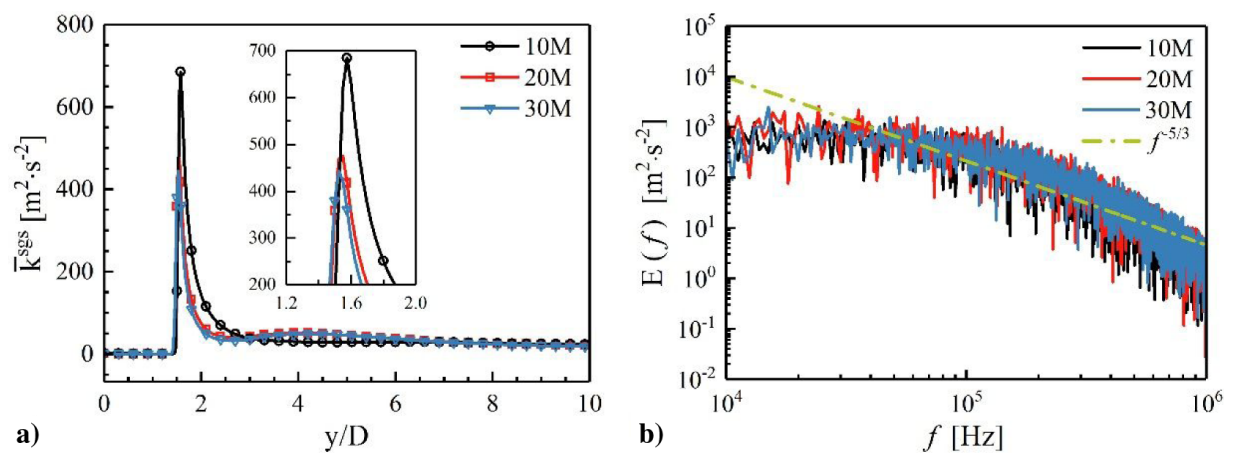

Fig. 6 Representations of a) time-averaged axial SGS turbulent kinetic energy; and b) energy spectrum sampled at $x / D=-1, y / D=6$, and $z / D=0$ for the three mesh resolutions.

the accuracy and the computational cost, the results of the medium mesh are employed in the following analysis.

\section{B. Time-Averaged Flow Characteristics}

Mean flow structures are presented first to give a general idea of how the vortex excitation modulates the highly underexpanded jet. Figure 7 shows the time-averaged contours of the Mach number, pressure, temperature, and density gradient in the quasi-steady stage for the free and excited jets. As shown in Fig. 7a1, the free jet is issued sonically from the nozzle, forming an expansion fan, and then rapidly expands to a Mach number around 3.0 before the Mach disk. Before the Mach disk, the corresponding temperature and pressure are reduced to the local minima, i.e., $\bar{T}_{\text {min }} \approx 120 \mathrm{~K}$ and $\bar{p}_{\text {min }} \approx 0.015 \mathrm{MPa}$, respectively. The vortex-excited jet undergoes more drastic expansion due to the low-pressure suction effect, rendering a much wider and bent Mach disk in Fig. 7d2. The Mach number behind the Mach disk is higher for the excited jet; correspondingly, the postshock pressure and temperature rise ratios are lower, implying that the vortex excitation weakens the strength of the Mach disk.

Table 2 compares the height $H_{m}$ and diameter $D_{m}$ of the Mach disk with data from the literature. A widely used empirical formula was derived by Ashkenas and Sherman [44] to describe the relationship between $H_{m}$ and the NPR as

$$
\frac{H_{m}}{D}=C_{H} \sqrt{\frac{p_{0}}{p_{\infty}}}
$$

where $C_{H}=0.67$ is considered to be constant when the NPR is between 20 and 200. Crist et al. [45] experimentally derived a similar constant $C_{H}=0.65$, which is suggested to be the most fitted value in the review by Franquet et al. [4]. For the LES modelings with lower NPRs of 5.50 [46] and 5.60 [7], the value of $C_{H}$ is around 0.60, which is close to the current predicted value of 0.59 . The normalized diameter $D_{m} / D$ of the excited jet is much larger than that of the free jets.

Not only the Mach barrel but also the subsequent wave structures are significantly modulated, as shown in Fig. 8. For the free jet, a train of shock cells is formed behind the Mach disk as denoted by the intense pressure and velocity oscillations that, however, are not observed in the excited jet. For the excited jet, the pressure and the velocity only experience slight variations before asymptotically reaching the far-field conditions. Note that the strengthened shear stress by the counterflow slows down the axial velocity of the excited jet behind the Mach disk.

\section{Mixing Enhancement Analysis}

The mixing area $A$ is used to quantify the mixing level and is defined as the contour area between $Y_{N 2}=0.77165$ and $Y_{N 2}=0.99534$, corresponding to the mixture fractions of $X=0.02$ and $X=0.98$. This definition stems from previous studies [8], and it indicates that the jet/air mixing still takes place at the jet boundary of $X=0.02$ and air boundary of $X=0.98$, whereas can be ignored beyond the boundaries. Thus, a larger mixing area means a wider diffusion range, and thus a higher mixing level.

Figure 9 shows the streamwise variation of time-averaged mixing area normalized by the nozzle exit area $A_{e}$. For $y / D \leq 4$, the reduction in the mixing area in the excited jet is due to the leakage of nitrogen through the collar exit caused by the entrainment of the 

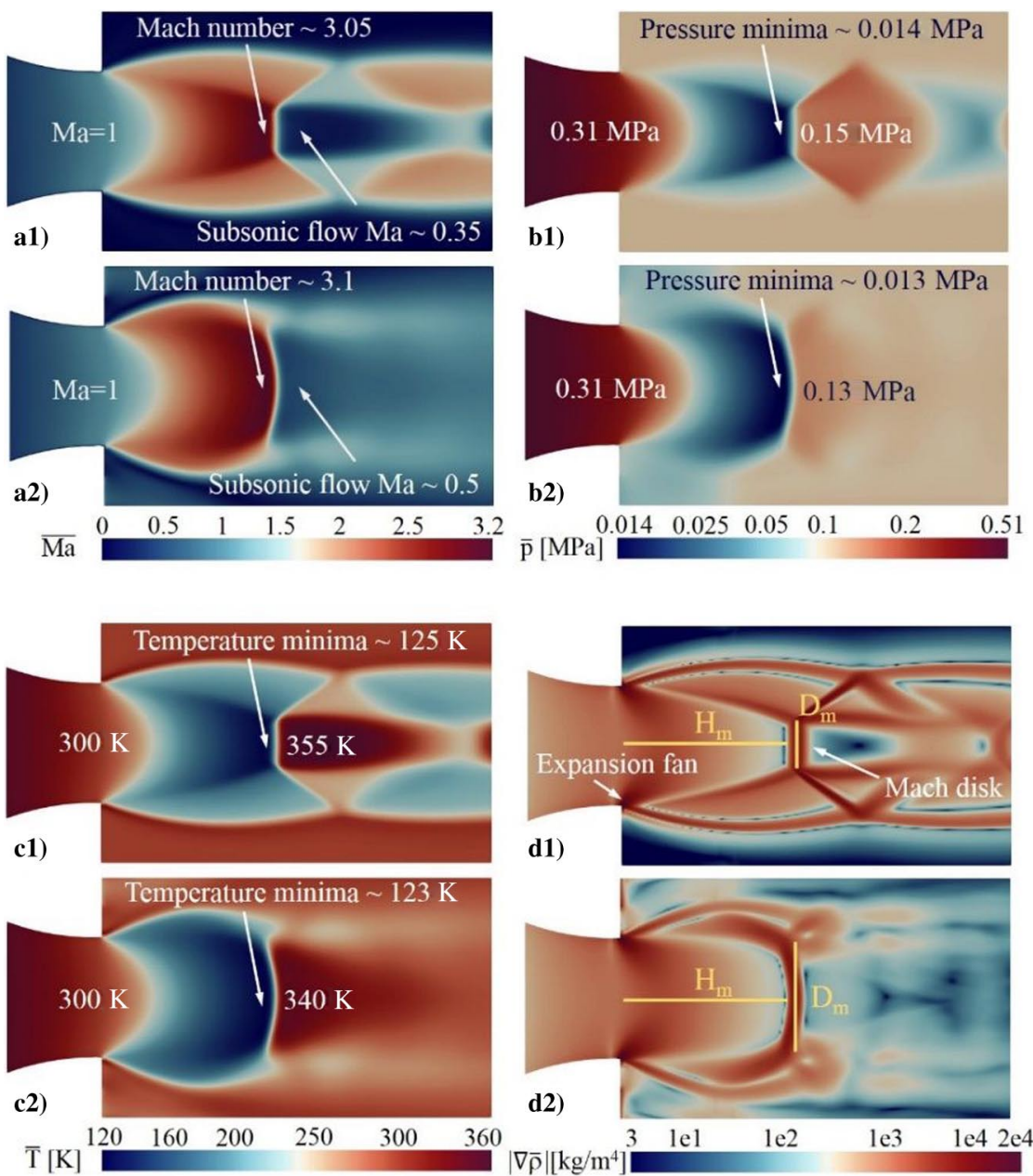

Fig. 7 Time-averaged a) Mach number, b) pressure, c) temperature, and d) density gradient on the centerplane $(z / D=0)$ for the a1-d1) free and a2-d2) excited jets.

Table 2 Height and diameter of Mach disk for highly underexpanded jets

\begin{tabular}{|c|c|c|c|c|c|c|}
\hline Reference & Method & $D, \mathrm{~mm}$ & $p_{0} / p_{\infty}$ & $H_{m} / D$ & $C_{H}$ & $D_{m} / D$ \\
\hline Ashkenas and Sherman [44] & Experiment & -- & $20 \sim 200$ & -- & 0.67 & -- \\
\hline Crist et al. [45] & Experiment & $0.66 \sim 3.02$ & $20 \sim 200$ & -- & 0.65 & -- \\
\hline Vuorinen et al. [46] & LES & 1.4 & 5.50 & 1.43 & 0.61 & 0.36 \\
\hline Li et al. [7] & LES & 2.0 & 5.60 & 1.43 & 0.60 & 0.38 \\
\hline Current free jet & LES & 2.0 & 5.60 & 1.39 & 0.59 & 0.38 \\
\hline Current excited jet & LES & 2.0 & 5.60 & 1.39 & 0.59 & 0.88 \\
\hline
\end{tabular}

counterflow. An average mass flow rate of $12.6 \%$ main inlet nitrogen is leaked through the collar exit.

For $6 \leq y / D \leq 18$, the jet/air mixing has been remarkably enhanced after imposing vortex excitation. The mixing area of the excited jet has a minimum increase of $10 \%$ at $y / D=6$ and a maximum of $200 \%$ at $y / D=12$. The improvement of overall mixing levels at the middle segment of the jet is attributed primarily to the augmented mutual interaction between vortical structures in the
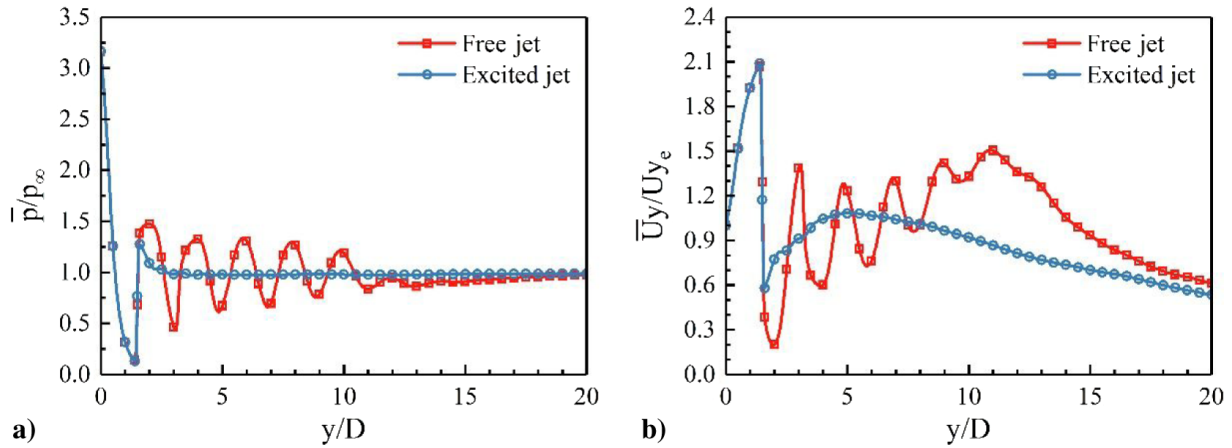

Fig. 8 Time-averaged axial a) pressure and b) streamwise velocity for the free and excited jets. 


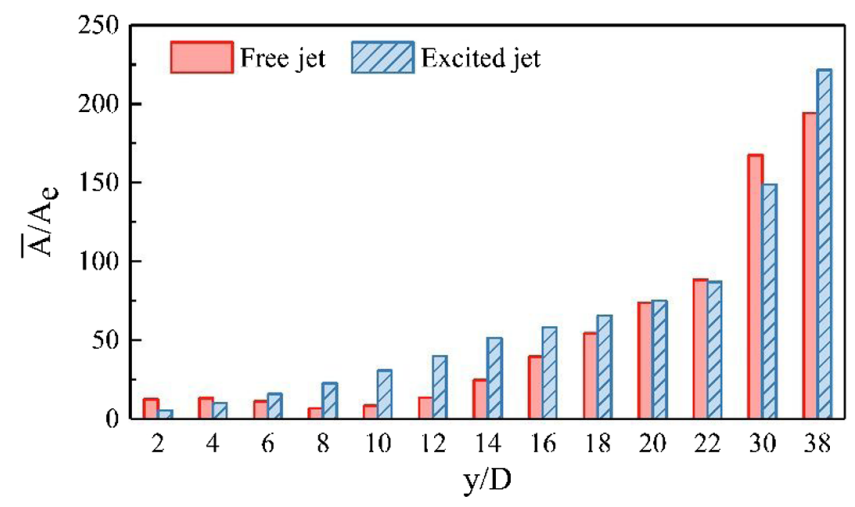

Fig.9 Time-averaged mixing area normalized by the nozzle exit area $A_{e}$ from $y / D=2$ to 38 .

jet shear layer and environment. In detail, as compared in Fig. 10, the hoop vortex in the excited jet, considered as the source of vortex excitation, continues to induce more vortices to be generated in the shear layer, which intensifies the K-H instability and the oscillation level of the shear layer, and then quickly breaks up the jet boundary to achieve large-scale interaction with the environment. Besides, the mixing enhanced region by vortex excitation is much wider compared with the enhanced region of $8 \leq y / D \leq 12$ by acoustic excitation in previous research [8].

Further downstream, after $y / D=22$ in Fig. 9, the difference of mixing levels between both jets is gradually reversed because the influence of the upstream vortex excitation diminishes to a dispensable level, which has little modulation on the mixing efficiency. At the further region near the outlet (e.g., $y / D=38$ ), jets have lower streamwise momentum, and thus are easier to affect by the boundary. Therefore, no obvious rule can be concluded for the influence on mixing area by the vortex excitation.

In addition to the enhanced overall mixing level, the underexpanded jet subjected to vortex excitation experiences a faster mixing process. The variation of mixing areas at the middle location of $y / D=10$ is illustrated in Fig. 11. Due to the slowed-down axial velocity, the excited jet takes a slightly longer time of $52 t_{0}$ to reach $y / D=10$ from the nozzle exit. However, it arrives at the peak of mixing within $16 t_{0}$, which is only half of the time taken by the free jet. This justifies that vortex excitation accelerates the starting mixing process in the middle jet. Furthermore, the mean mixing speeds of

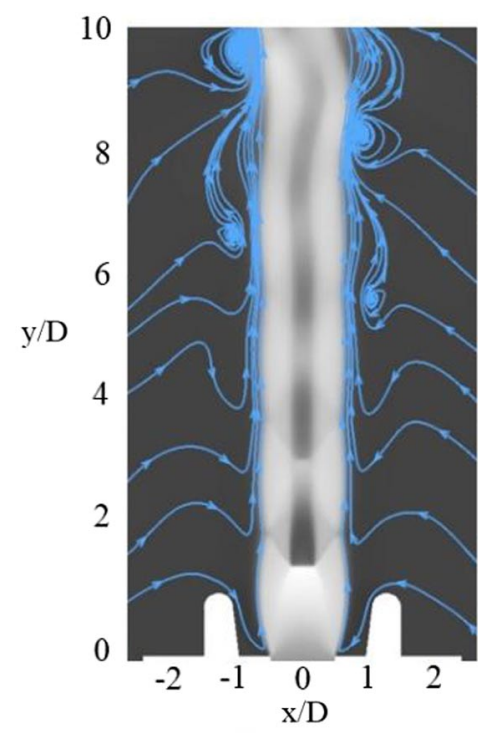

a) Free jet

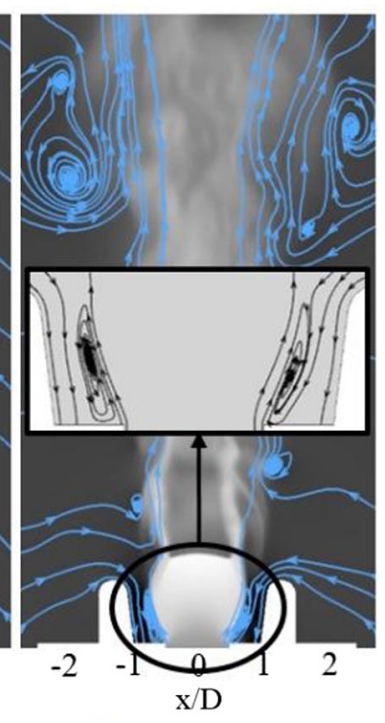

b) Excited jet
Fig. 10 Instantaneous streamwise velocity superposed with streamlines on the centerplane.

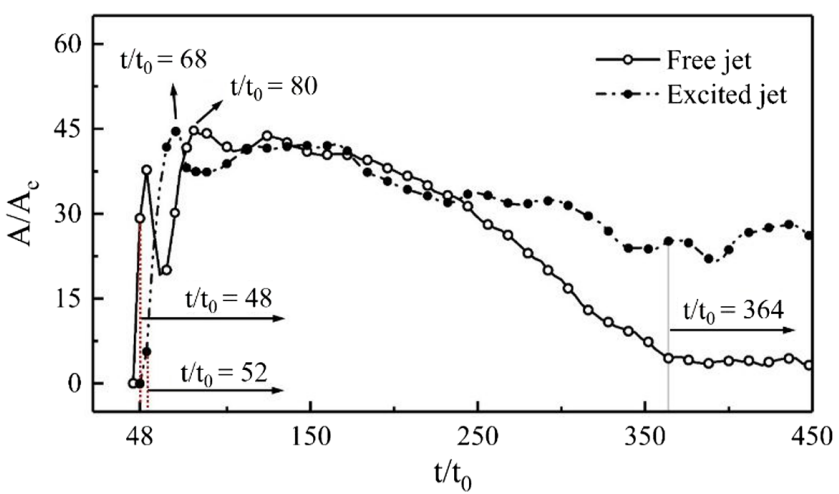

Fig. 11 Initial evolution of mixing areas at $y / D=10$.

jets can be characterized by their mixing volumes from $t=48 t_{0}$ to $t=364 t_{0}$; after which, both jets keep at each quasi-steady mixing level. The mixing volume is defined by the envelope area of each curve in Fig. 11 over the corresponding period of time. Thus, the visually higher mixing volume outlined by excited jet indicates that an average faster mixing process is achieved. Note that similar results can be obtained at the middle jet of $6 \leq y / D \leq 18$.

\section{Unsteady Evolution of Flow Structures}

Temporal evolutions of shock waves and vortex structures are analyzed here to show the unsteady features' modulations by vortex excitation on a typical highly underexpanded jet, including shock formation, shock/shear-layer interaction, vortex evolution, the jet destabilization process, and turbulence transition. Considering their significant roles in the mixing process, the analysis helps to interpret the mixing enhancement mechanism.

\section{Wave Structures}

As shown in Fig. 12, a bow shock is formed at the early time of $t=2 t_{0}$, and then it propagates spherically. At this stage, the vortex excitation has minimal influence because the hoop vortex has not been fully established. In the free and excited jets, with a fairly high degree of underexpansion (say, $p_{e} / p_{\infty}=4$ in this study, where $p_{e}$ is the pressure at the nozzle exit), the subsonic jet is quickly accelerated to be supersonic once it is issued from the nozzle orifice, and it is accompanied by a series of Prandtl-Meyer (P-M) expansion waves at $t=8 t_{0}$. Then, at $t=16 t_{0}$, the $\mathrm{P}-\mathrm{M}$ expansion waves reflect at the jet boundary and form compression waves, which then merge into the barrel shock and the Mach disk. The formation of the Mach disk is the indicator of a highly underexpanded jet [42]. Meanwhile, an oblique shock (i.e., reflected shock) emanates from the triple point; a slip line forms between the supersonic flow and the subsonic flow, downstream of the oblique shock and the Mach disk, respectively.

For the free jet, at $t=24 t_{0}$, the subsonic core region behind the Mach disk is once again accelerated into the supersonic flow, which forms the second normal shock. Similarly, at $t=32 t_{0}$, the third and subsequent shock cells are formed, resembling the previous one. The total pressure of the potential jet decays after each normal shock wave, ending with a series of oblique shock waves. In the meantime, the $\mathrm{K}-\mathrm{H}$ instability arises and rolls the shear layer to form vortex rings, which then perturb the shock waves to exhibit small-amplitude periodic oscillations. At $t=40 t_{0}$, the shock/shear-layer reflection and impingement establish the typical structures of a highly underexpanded jet $[\underline{4}, \underline{47}, \underline{48}]$.

However, for the excited jet, due to a wider width of Mach disk caused by the suction, the gap between slip lines becomes too large for the oblique shocks to form a closed shock cell after the Mach disk. Instead, the oblique shocks interact with the slip line and the jet boundary; between which, a few small oblique shocks are formed at $t=32 t_{0}$. Later, the intensified K-H instability significantly destabilizes the shocks and the shear layer after the Mach disk, accordingly boosting their mutual interaction, which contributes to a rapid nearfield destabilization process, and thus a faster mixing. 

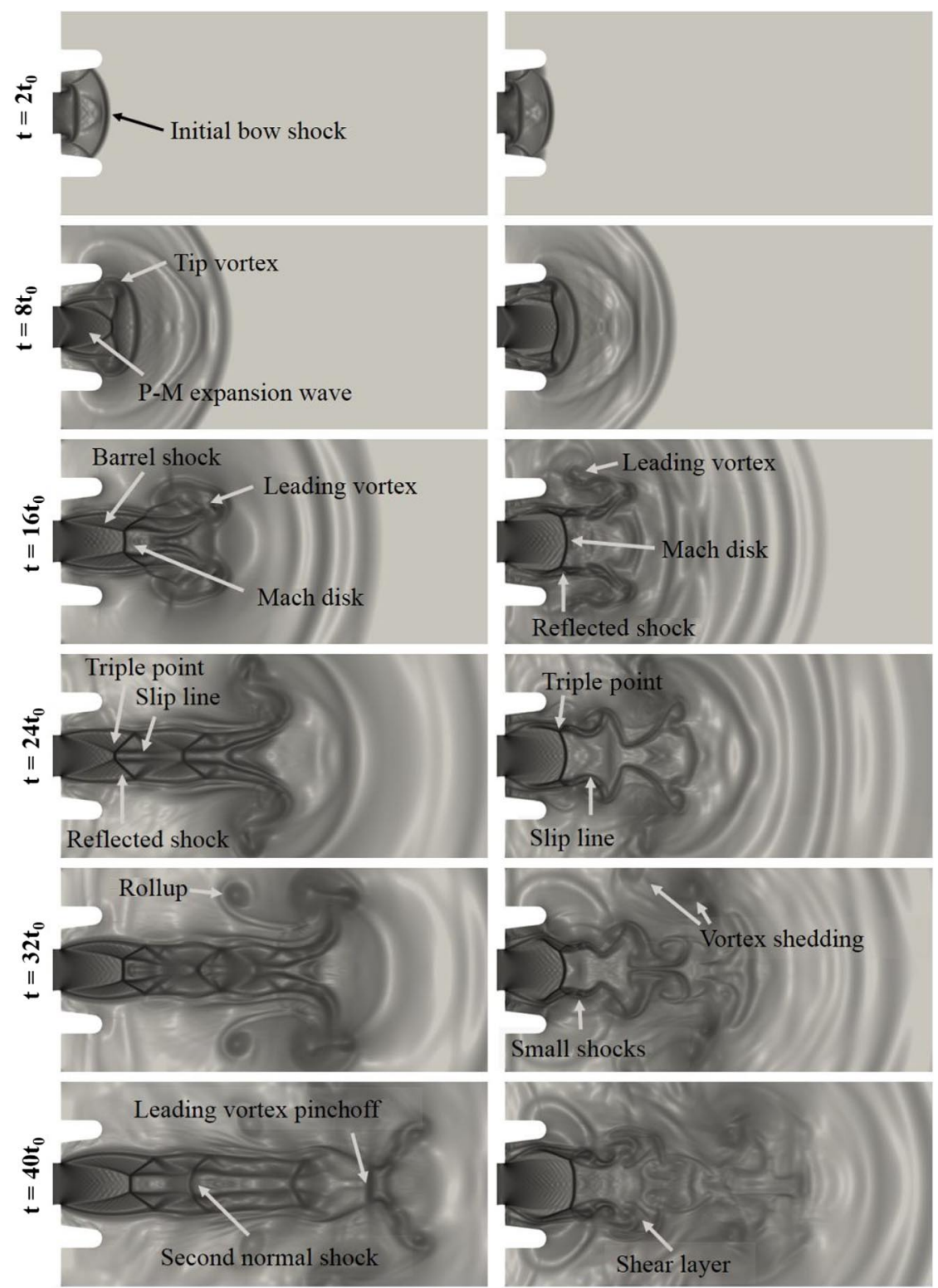

a) Free jet

b) Excited jet

Fig. 12 Time evolution of wave structures on the centerplane.

\section{Vortex Structures}

Figure 13 compares the evolution of vortex structures represented by the $\mathrm{Q}$ criterion for the free and excited jets. Here, the $\mathrm{Q}$ criterion is defined as

$$
Q=\frac{1}{2}\left(\Omega_{i j} \Omega_{i j}-S_{i j} S_{i j}\right)
$$

where $\Omega_{i j}$ and $S_{i j}$ denote the rotation tensor and strain tensor, respectively. Here, the isosurface of $Q=3 \times 10^{7} \mathrm{~s}^{-2}$ is adopted and colored by the streamwise velocity. In the early time before $t=24 t_{0}$, a recirculation zone, caused by the obstruction of the initial quiescent environment, is formed upon tips in free and excited jets. For the free jet, the recirculation zone breaks up from the center and falls off to form undulating vortex rings at $t=48 t_{0}$, which parallelly wrap around jet core. The vortex rings are inclined to distort when the transition begins at $t=84 t_{0}$. Meanwhile, streamwise vortex filaments stretch from the topmost vortex ring. Later, at $t=128 t_{0}$, the upstream vortex rings are tilted and distorted by the $\mathrm{K}-\mathrm{H}$ instability, forming into hairpin vortices with counter-rotating legs connecting to the jet core, which destabilizes the jet core and causes large-scale mass diffusion and mixing. At $t=200 t_{0}$, the circumferential instability promotes the formation of the large-scale $\Omega$-shape vortices downstream, which is also indispensable for the mixing [10]. When the turbulence is fully developed at $t=400 t_{0}$, initial undulating rings vanish, leaving only helical vortices around the jet core.

Regarding the excited jet, the turbulence transition occurs earlier at $t=64 t_{0}$. From $t=84 t_{0}$, the upstream vortex rings are directly broken into finer vortices by the intensified $\mathrm{K}-\mathrm{H}$ instability with the vortex excitation. There are less vortex filaments emanating from the tip rings; instead, more outward-tilting hairpin vortices emerge. The dramatically increased number of deformed vortices in the excited jet accelerates and intensifies the jet destabilization process, leading to a faster and stronger diffusion and mixing process. Exactly from the time of $t=128 t_{0}$, the hairpin vortices merge with the detached vortices from the tip, which enhances the formation and development of the large-scale $\Omega$-shape vortices. At the quasi-steady state of $t=400 t_{0}$, there are obviously richer fine vortices in the middle of the excited jet, contributing to a significantly higher mixing level.

Vorticity analysis is conducted to reveal the mechanism of vorticity generation and destruction with the modulation by the vortex excitation. The compressible vorticity transport equation is used to describe the vorticity evolution: 
a)
1) $\mathrm{t}=8 \mathrm{t}$
2) $t=24 t_{0}$
3) $t=36 t_{0}$
4) $t=48 t_{0}$
5) $t=64 t_{0}$
6) $\mathrm{t}=84 \mathrm{t}_{0}$
7) $t=128 t_{0}$
8) $t=200 t_{0}$
9) $t=400 t_{0}$
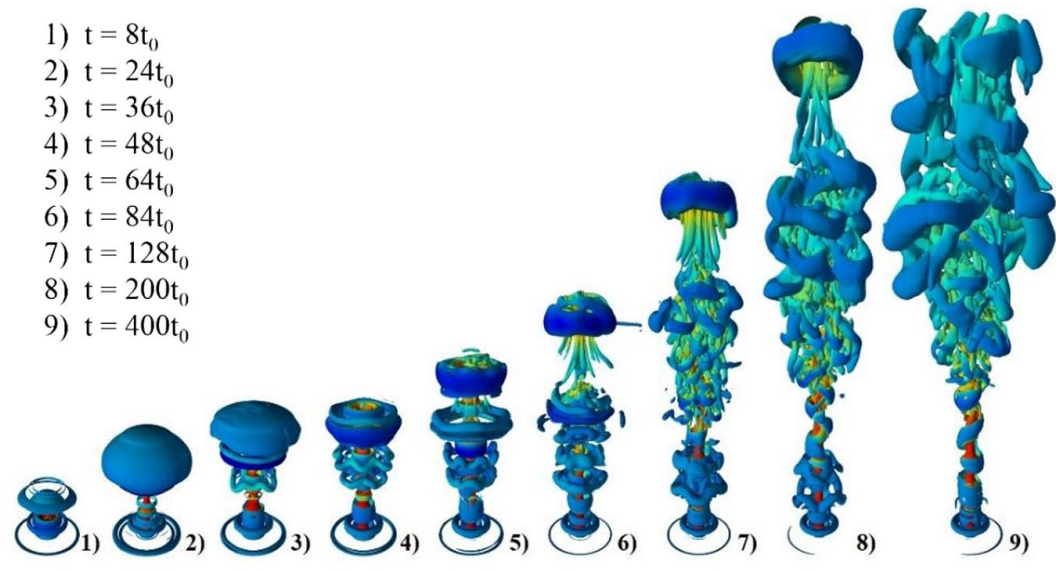

$\mathrm{Uy}[\mathrm{m} / \mathrm{s}]$

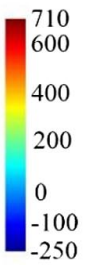

b)
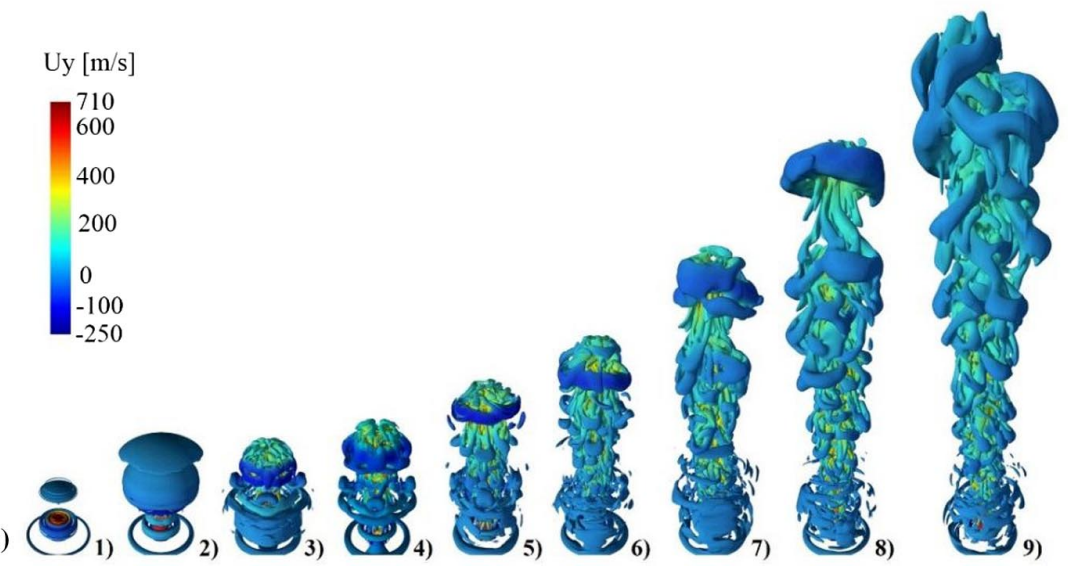

Fig. 13 Three-dimensional instantaneous isosurfaces of $Q$ criterion $\left(Q=3 \times 10^{7} \mathrm{~s}^{-2}\right)$ colored by streamwise velocity $U y$ for the a) free and b) excited jets.

$$
\begin{aligned}
\frac{\partial \boldsymbol{\omega}}{\partial t} & =\underbrace{-(\boldsymbol{U} \cdot \nabla) \boldsymbol{\omega}}_{\text {conv }}+\underbrace{(\boldsymbol{\omega} \cdot \nabla) \boldsymbol{U}}_{V S} \underbrace{-\boldsymbol{\omega}(\nabla \cdot \boldsymbol{U})}_{\text {dila }}+\underbrace{\left(1 / \rho^{2}\right) \nabla \rho \times \nabla p}_{\text {baro }} \\
& +\underbrace{\nu \nabla^{2} \boldsymbol{\omega}}_{\text {diff }}+\underbrace{\nabla(\nu) \times\left[(1 / 3) \nabla(\nabla \cdot \boldsymbol{U})+\nabla^{2} \boldsymbol{U}\right]}_{\text {VG }}
\end{aligned}
$$

The annotations of conv, VS, dila, baro, diff, and VG correspond to the convective, vortex stretching, dilatational, baroclinic, diffusive, and viscosity gradient terms, respectively.

In Fig. 14, the convective term has a higher magnitude in the core region for both the free and excited jets because it is proportional to the local velocity and vorticity. It is increased by an order of magnitude in the near-nozzle region of the excited jet. The vortex stretching term is regarded as the generation source of small vortices through stretching and curving the vortex tubes into fine ones, accompanied by the turbulence energy cascade. Therefore, the high VS magnitude focuses in the region of $10 \leq y / D \leq 15$ for the free jet and $y / D \leq 5$ for the excited jet, where the small vortices are increased with the instability developing in shear layer. The vortex excitation magnifies the VS term by at least two orders of magnitude in the near-nozzle region, which is probably attributed to the stretching force imposed on the vortices by the external suction. This can contribute to a local sufficient mixing. To quantitatively support this finding, the area integrals of time-averaged vorticity terms in Eq. (14) on different transverse planes are included as Supplemental Fig. S1. The dilatational term represents the contribution of the volume change of fluid lumps, which is closely related to the compressibility; thus, it peaks in the zone where shock waves reside. Based on the conservation law of angular momentum, the dilatational term is neither a source nor a sink of vortices; it just redistributes the vorticity according to the strength of compressibility. Hence, the vanished shocks after the Mach disk in the excited jet reduce the overall magnitude of the dilatational term, except in the near-nozzle region. The baroclinic term, which also peaks in the regions with rapid expansion and strong compression, is weakened when the vortex excitation is imposed. The diffusion term describes the viscous diffusion of vorticity and is therefore higher in the mixing layer where high turbulent viscosity exists. Because the diffusion term can be nondimensionalized into $R e^{-1} \nabla^{2} \omega$, the overall high Reynolds number in the supersonic jet flow leads to a negligible viscosity diffusion effect, which has the lowest order of magnitude among all terms. The last term related to viscosity gradient, defined as VG in this work, is not reported here. Its maximum values, which are of the same order with convective term, are at shock waves, and they are increased by an order in the near-nozzle region of the excited jet.

\section{E. Dominating Instability Mode}

A distinct feature of highly underexpanded jets is the helical instability mode $[\underline{7,49}, \underline{50}]$. Previous research showed that the helical modes vary from different NPRs, and a single helical mode is dominant in the jet with NPR $=5.60$ [7]. To visualize the helical mode, the concept of local helicity $H$ is employed:

$$
H=\omega \cdot \boldsymbol{U}
$$

which interprets the correlation between vorticity and velocity. Note that the positive and negative signs of $H$ represent two opposite directions of local helical motions; i.e., $H>0$ indicates clockwise, $H<0$ indicates counterclockwise, and $H \rightarrow 0$ indicates nonhelical. The helicity distribution visualizes the spatial structure of coherent helical vortices in the shear layer; and the temporal evolution of helicity shows the large-scale motions of those coherent structures, including entanglement, rotating, and transporting, which characterizes the jet instability mode.

From Fig. 15a, the helices in the near field of the free jet form a single helical mode before $y / D \leq 8$, which consists of a pair of branches with opposite signs of helicity, indicating that the two branches have locally clockwise and counterclockwise helical motions, respectively. Besides, the superposition of the helical branches forms the overall motion of the 

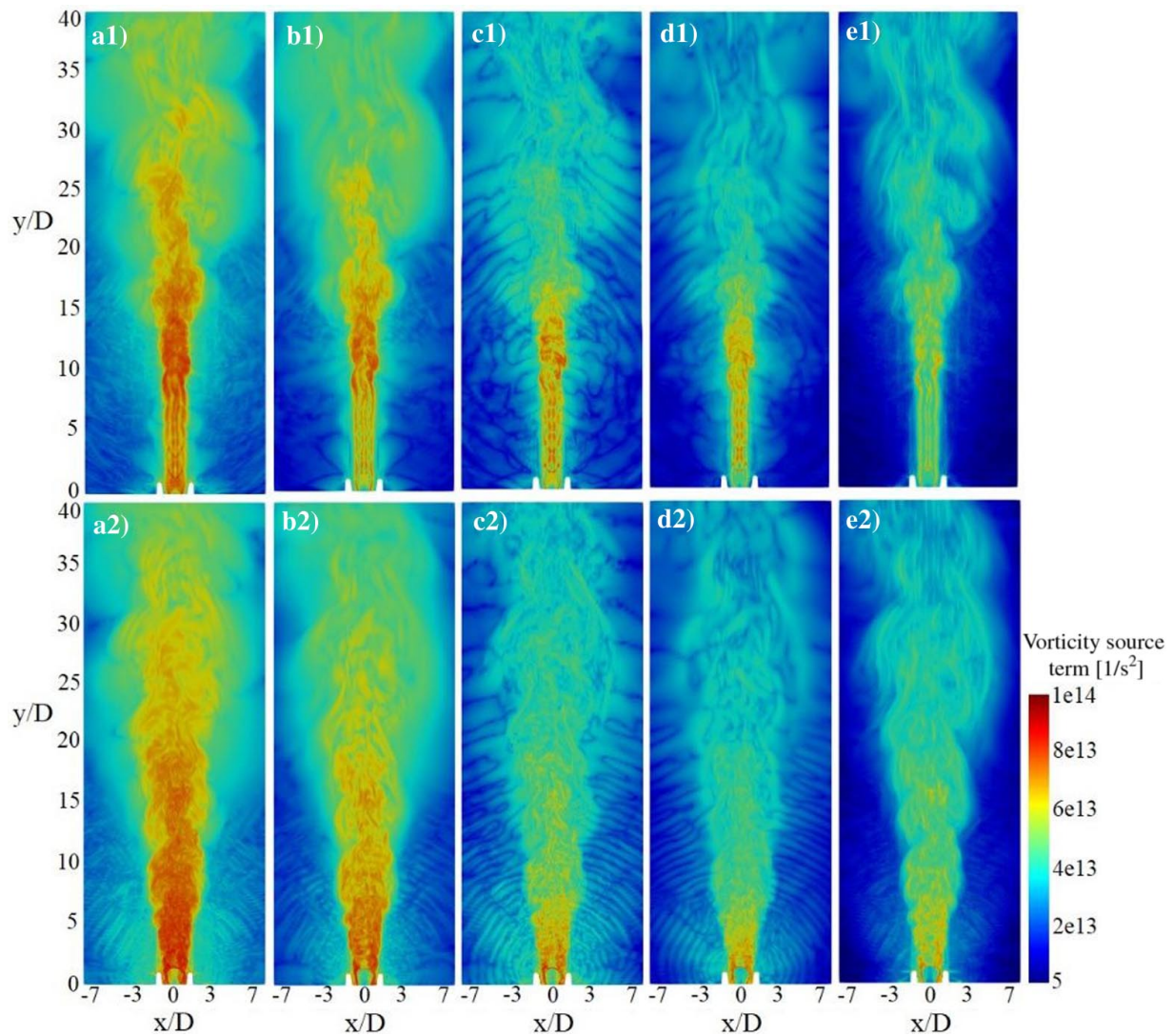

Fig. 14 Magnitudes of instantaneous a) convection, b) vortex stretching, c) dilatation, d) baroclinic, and e) diffusion terms on the centerplane for the a1-d1) free and a2-d2) excited jets.

shear layer, which rotates clockwise and counterclockwise alternately with jet evolution. The single helical mode is similar to the antisymmetric helical mode with an azimuthal wave number of $m=1$ found in underexpanded jets [49-51] and swirling jets [52,53]. The three-dimensional structure of the single helical mode in free jet is shown in Fig. 16a, where the braidlike helical structure showing a sense of winding can be clearly observed in $4 \leq y / D \leq 8$. However, beyond $y / D=15$, the helical structures break up into chaotic segments with the development of hydrodynamic instability.

For the excited jet, due to the enhanced disturbances in the shearlayer coherent structures by the vortex excitation, the local motions of helical branches are intensified to generate a higher order helical mode with a larger azimuthal wave number at $y / D=2$. The higherorder helical mode at $y / D=2$ consists of uncountable pairs of entangled helical branches with opposite local helical directions, as shown in Fig. 15b, and this special pattern is defined here as multiple helical mode. The overall rotation of the helical structures is accelerated by the vortex excitation but also in clockwise and counterclockwise directions alternately. Figure $16 \mathrm{~b}$ displays the spatial correlation of the multiple helical structures with mixed clockwise and counterclockwise helicoids in the region $y / D<4$. Further at $y / D \geq 4$, vortex excitation accelerates the broken process

a)

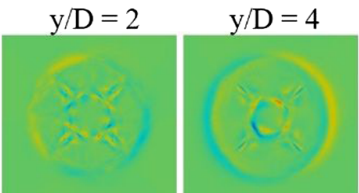

$\mathrm{y} / \mathrm{D}=6$

$\mathrm{y} / \mathrm{D}=8$
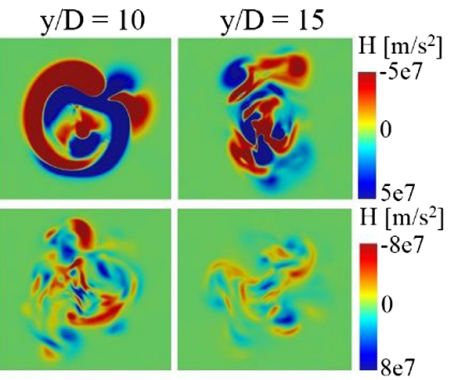

b)
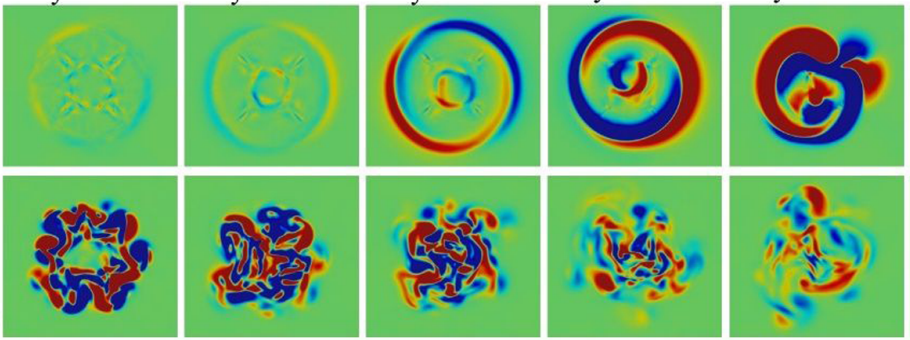

Fig. 15 Quasi-steady helicity on transverse planes of the a) free and b) excited jets.
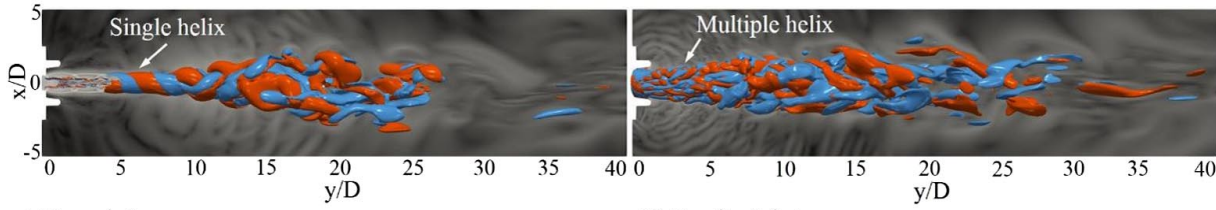

a) Free jet

b) Excited jet

Fig. 16 Three-dimensional isosurfaces of helicity $\left(H= \pm 10^{7} \mathrm{~m} \cdot \mathrm{s}^{-2}\right)$ on the background of the density gradient. 


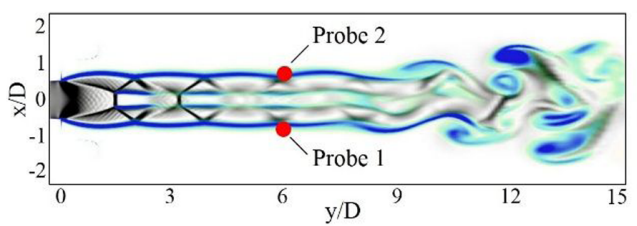

a) Free jet

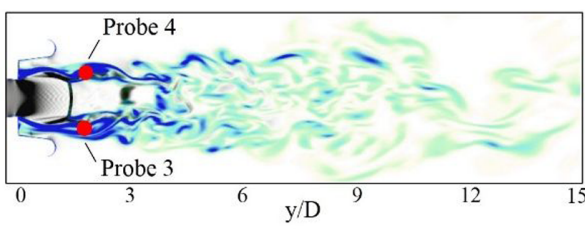

b) Excited jet

Fig. 17 Locations of probes for fluctuation measurement shown in instantaneous vorticity magnitude contours superposed on wave structures: a) free jet and b) excited jet.

of the helical structures, rendering an intricate flow pattern similar to the free jet at $y / D=15$.

To quantify the jet instability mode controlling the helical structures, pressure fluctuations on either side of the jet shear layer are traced for the free and excited jets. Given that the helical modes in the shear layer are clearly identified at $y / D=6$ for the free jet and $y / D=2$ for the excited jet, as shown in Fig. 15, the sampling probes are set at the corresponding locations, as displayed in Fig. 17. In Fig. $17 \mathrm{a}$ (representing the free jet), the values are $x / D \bar{\equiv}-1$, $y / D \overline{=6}$, and $z / D=0$ for probe 1 and $x / D=1, y / D=6$, and $z / D=0$ for probe 2. In Fig. $17 \mathrm{~b}$ (representing the excited jet), the values are $x / D=-1, y / D \overline{=2}$, and $z / D=0$ for probe 3 and $x / D=1, y / D=2$, and $z / D=0$ for probe 4 . The signals are analyzed using the fast Fourier transformation method to obtain frequency and phase spectrums. Similar analyses $[43,54,55]$ were conducted on pressure fluctuations to study the instability modes.

The spectrums of free jet are presented in Fig. 18, which shows that frequency spectrum on either side of jet is generally matched, and the amplitude peaks at $f_{s}=41.3 \mathrm{kHz}$, corresponding to the fundamental screech tone frequency. The spectrums agree well with that of the underexpanded jet of NPR $=5.60$ given in previous research [7].
The phase difference on either side of the jet is $-177.2 \mathrm{deg}$, which is very close to $180 \mathrm{deg}$, representing an antisymmetric mode with sinuous instability, which was also observed in the work of Berlans et al. [54] and Raman and Rice [55]. This antisymmetric mode corresponds to the single helical mode generated in the free jet. The other peaks at higher frequencies, which have a decreasing amplitude with frequency increases, are harmonics of the screech tone, such as $f=50.6$ and $54.6 \mathrm{kHz}$ shown in Fig. 18; and they are approximately $180 \mathrm{deg}$ out of phase on either side of the free jet. In addition, the phase difference of zero, which represents a symmetric mode with varicose instability, is observed in their works [54,55] but not found here in dominant frequencies at $y / D=6$, which demonstrates that the symmetric mode is not the dominant instability mode in the fully developed underexpanded jet.

Figure 19 outlines spectrums of signals in the excited jet. Increased amplitudes of screech tone frequencies are observed in the excited jet compared with the free jet, indicating a more intense screech tone in the excited jet. It is attributed to a stronger interaction between instability waves and shock waves in the excited jet, which is achieved by the vortex excitation through intensifying the oscillation of coherent structures in shear layer. The stronger jet/air interaction also

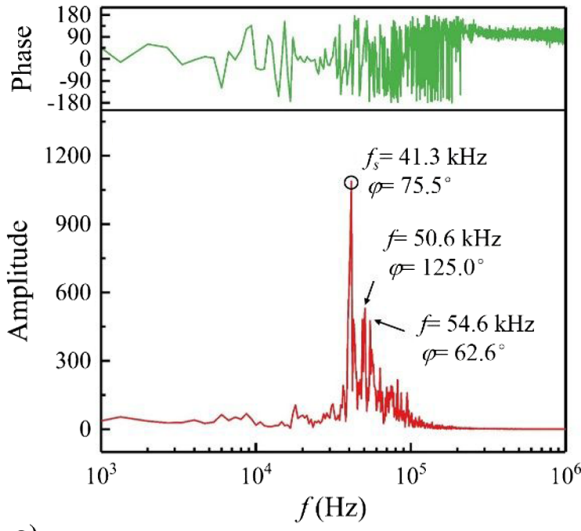

a)

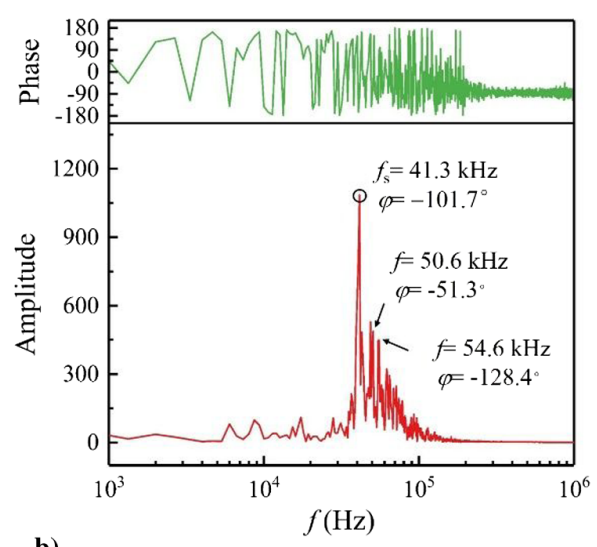

b)

Fig. 18 Frequency and phase spectrums of pressure fluctuations on either side of the jet shear layer at a) probe 1 and b) probe 2 for the free jet.
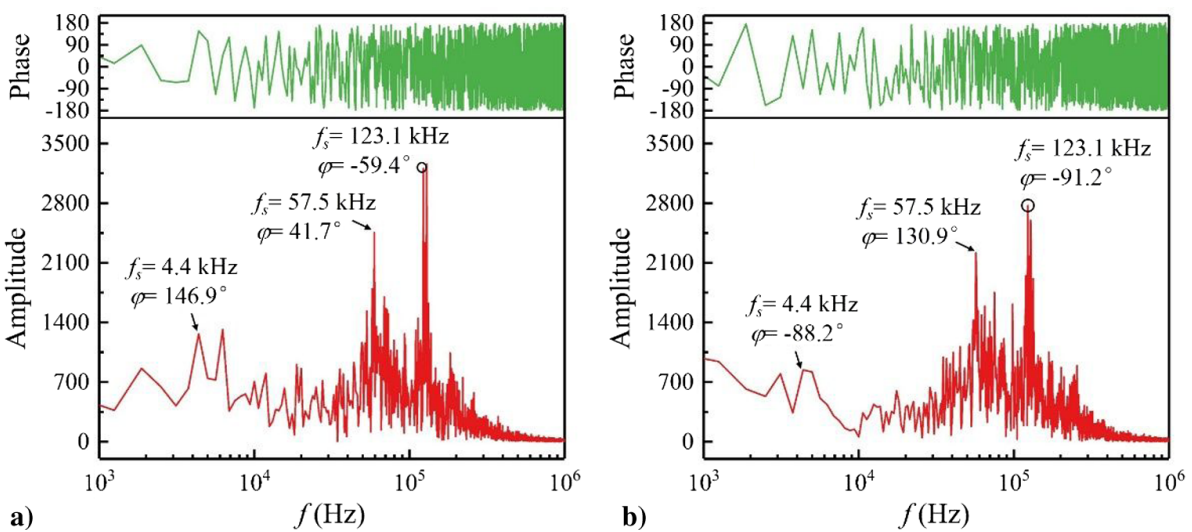

Fig. 19 Frequency and phase spectrums of pressure fluctuations on either side of the jet shear layer at a) probe 3 and b) probe 4 for the excited jet. 
enhances the mixing performance. Besides, the frequency of the fundamental screech tone is $f_{s}=123.1 \mathrm{kHz}$, which is approximately three times higher than that for the free jet. This increase is mainly modulated by the disappeared shocks downstream through considerably shortening the loop distance. The phase difference of $f_{s}$ on either side of the jet is also modulated by the enhanced instability-wave/ shock interaction, which is neither 0 nor $180 \mathrm{deg}$, indicating that the dominant mode is asymmetric and is identical to the higher-order multiple helical mode at $y / D=2$ shown in Fig. 15b. Besides, lower frequencies such as 57.5 and $4.4 \mathrm{kHz}$ are more excited compared with the higher frequencies, and their phase differences prove that multiple helical modes are dominant in the excited jet shear layer at $y / D=2$.

\section{Conclusions}

In this study, large-eddy simulation modelings of a highly underexpanded jet with a nozzle pressure ratio of 5.60 and the same jet subjected to a vortex excitation are conducted to reveal the modulation of vortex excitation on flow characteristics and mixing enhancement. The main conclusions are summarized as follows:

The vortex excitation has distinctly improved the mixing efficiency of the injection nitrogen with the environment, shown by a considerable increase of the mixing level and a faster mixing process in the middle jet. What directly contributes to the remarkable enhancement is the augmented mutual interaction between vortical structures in the jet shear layer and environment.

The vortex excitation also modulates the establishment process of the near-field wave structures. Only the first shock cell survives in the excited jet, including the P-M expansion wave, Mach disk, and barrel shock; but, these structures are generated with different locations and shapes from the free jet. The intensified $\mathrm{K}-\mathrm{H}$ instability produces a stronger shock/shear-layer interaction, which contributes to a rapid near-field destabilization and mixing process.

The transition process of turbulence is also accelerated by the vortex excitation, shown by remarkably increased and distorted hairpin vortices as well as the prearrival large-scale $\Omega$-shape vortices. Quantitatively, all contributions to vorticity generation are amplified by at least an order in the near field, of which the vortex stretching term is far ahead, contributing to a local sufficient mixing.

The antisymmetric single helical mode with an azimuthal wave number of $m=1$ in the highly underexpanded jet is modulated into an asymmetric higher-order multiple helical mode with a larger wave number in the very near field of the excited jet, which corresponds to different pressure fluctuation spectrums in the jet shear layer. The vortex excitation remarkably excites the shear-layer oscillation, and thus the interaction between coherent structures and shocks, to generate a significantly more intense screech tone.

\section{Acknowledgments}

The present study was supported by National Natural Science Foundation of China (grant no. 11502270) and the Training Program of the Major Research Plan of the National Natural Science Foundation of China (grant no. 91641110). The authors are also grateful to the National Supercomputer Center of Tianjin for providing the computational resource and Xi Xia for providing important advice based on this work.

\section{References}

[1] Adamson, T. C., Jr., and Nicholls, J. A., "On the Structure of Jets from Highly Underexpanded Nozzles into Still Air," Journal of the Aerospace Sciences, Vol. 26, No. 1, 1959, pp. 16-24.

https://doi.org/10.2514/8.7912

[2] Seiner, J. M., Dash, S. M., and Kenzakowski, D. C., "Historical Survey on Enhanced Mixing in Scramjet Engines," Journal of Propulsion and Power, Vol. 17, No. 6, 2001, pp. 1273-1286. https://doi.org/10.2514/2.5876

[3] Northam, G., Greenberg, I., and Byington, C., "Evaluation of Parallel Injector Configurations for Supersonic Combustion," 25th Joint Propulsion Conference, AIAA Paper 1989-2525, 1989.

[4] Franquet, E., Perrier, V., Gibout, S., and Bruel, P., "Free Underexpanded Jets in a Quiescent Medium: A Review," Progress in Aerospace Sciences,
Vol. 77, Aug. 2015, pp. 25-53.

https://doi.org/10.1016/j.paerosci.2015.06.006

[5] Huang, W., "Transverse Jet in Supersonic Crossflows," Aerospace Science and Technology, Vol. 50, March 2016, pp. 183-195. https://doi.org/10.1016/j.ast.2016.01.001

[6] Huang, W., and Yan, L., "Progress in Research on Mixing Techniques for Transverse Injection Flow Fields in Supersonic Crossflows," Journal of Zhejiang University Science A, Vol. 14, No. 8, 2013, pp. 554-564. https://doi.org/10.1631/jzus.A1300096

[7] Li, X., Yao, W., and Fan, X., "Large-Eddy Simulation of Time Evolution and Instability of Highly Underexpanded Sonic Jets," AIAA Journal, Vol. 54, No. 10, 2016, pp. 3191-3211. https://doi.org/10.2514/1.J054689

[8] Li, X., Fan, E., Yao, W., and Fan, X., "Numerical Investigation of Characteristic Frequency Excited Highly Underexpanded Jets," Aerospace Science and Technology, Vol. 63, April 2017, pp. 304-316. https://doi.org/10.1016/j.ast.2017.01.005

[9] Tan, J., Zhang, D., and Lv, L., "A Review on Enhanced Mixing Methods in Supersonic Mixing Layer Flows," Acta Astronautica, Vol. 152, Nov. 2018, pp. 310-324. https://doi.org/10.1016/j.actaastro.2018.08.036

[10] Brown, G. L., and Roshko, A., "On Density Effects and Large Structure in Turbulent Mixing Layers," Journal of Fluid Mechanics, Vol. 64, No. 4, 1974, pp. 775-816. https://doi.org/10.1017/S002211207400190X

[11] Li, Q., Chen, H., and Fu, S., "Large-Scale Vortices in High-Speed Mixing Layers," Physics of Fluids, Vol. 15, No. 10, 2003, pp. 3240-3243. https://doi.org/10.1063/1.1602075

[12] Dimotakis, P. E., "Turbulent Free Shear Layer Mixing and Combustion," High-Speed Flight Propulsion Systems, AIAA, Reston, VA, 1991, pp. 265-340.

[13] Lieuwen, T. C., Unsteady Combustor Physics, Cambridge Univ. Press, New York, 2012, Chap. 2

[14] Murugappan, S., Gutmark, E., and Carter, C., "Control of Penetration and Mixing of an Excited Supersonic Jet into a Supersonic Cross Stream," Physics of Fluids, Vol. 17, No. 10, 2005, Paper 106101. https://doi.org/10.1063/1.2099027

[15] Mingbo, S., Hongbo, W., Jianhan, L., and Zhenguo, W., "Large Eddy Simulation of Mixing Enhancement of a 2-D Supersonic Mixing Layer Induced by Inflow Periodic Temperature Excitation," AIAA Paper 2009-7407, 2009. https://doi.org/10.2514/6.2009-7407

[16] Berglund, M., Fedina, E., Fureby, C., Tegnér, J., and Sabel'nikov, V., "Finite Rate Chemistry Large-Eddy Simulation of Self-Ignition in Supersonic Combustion Ramjet," AIAA Journal, Vol. 48, No. 3, 2010, pp. 540-550. https://doi.org/10.2514/1.43746

[17] Génin, F., and Menon, S., "Simulation of Turbulent Mixing Behind a Strut Injector in Supersonic Flow," AIAA Journal, Vol. 48, No. 3, 2010, pp. 526-539. https://doi.org/10.2514/1.43647

[18] Gruber, M. R., Baurle, R. A., Mathur, T., and Hsu, K. Y., "Fundamental Studies of Cavity-Based Flameholder Concepts for Supersonic Combustors," Journal of Propulsion and Power, Vol. 17, No. 1, 2001, pp. 146-153. https://doi.org/10.2514/2.5720

[19] Sislian, J., and Schumacher, J., "A Comparative Study of Hypersonic Fuel/Air Mixing Enhancement by Ramp and Cantilevered Ramp Injectors," AIAA Paper 1999-4873, 1999. https://doi.org/10.2514/6.1999-4873

[20] Gruber, M., Donbar, J., Jackson, T., Mathur, T., Eklund, D., and Billig, F., "Performance of an Aerodynamic Ramp Fuel Injector in a Scramjet Combustor," AIAA Paper 2000-3708, 2000. https://doi.org/10.2514/6.2000-3708

[21] Huang, W., Li, S., Yan, L., and Wang, Z., "Performance Evaluation and Parametric Analysis on Cantilevered Ramp Injector in Supersonic Flows," Acta Astronautica, Vol. 84, March 2013, pp. 141-152. https://doi.org/10.1016/j.actaastro.2012.11.011

[22] Li, L., Huang, W., Fang, M., Shi, Y., Li, Z., and Peng, A., "Investigation on Three Mixing Enhancement Strategies in Transverse Gaseous Injection Flow Fields: A Numerical Study," International Journal of Heat and Mass Transfer, Vol. 132, April 2019, pp. 484-497. https://doi.org/10.1016/j.ijheatmasstransfer.2018.12.038

[23] Huang, W., "Mixing Enhancement Strategies and Their Mechanisms in Supersonic Flows: A Brief Review," Acta Astronautica, Vol. 145, April 2018, pp. 492-500. https://doi.org/10.1016/j.actaastro.2018.02.022

[24] Strykowski, P. J., and Niccum, D. L., "The Stability of Countercurrent Mixing Layers in Circular Jets," Journal of Fluid Mechanics, Vol. 227, 
June 1991, pp. 309-343.

https://doi.org/10.1017/S0022112091000137

[25] Strykowski, P. J., and Wilcoxon, R. K., "Mixing Enhancement due to Global Oscillations in Jets with Annular Counterflow," AIAA Journal, Vol. 31, No. 3, 1993, pp. 564-570. https://doi.org/10.2514/3.11366

[26] Strykowski, P. J., Krothapalli, A., and Wishart, D., "Enhancement of Mixing in High-Speed Heated Jets Using a Counterflowing Nozzle," AIAA Journal, Vol. 31, No. 11, 1993, pp. 2033-2038.

https://doi.org/10.2514/3.11887

[27] Chuech, S., Lai, M.-C., and Faeth, G., "Structure of Turbulent Sonic Underexpanded Free Jets," AIAA Journal, Vol. 27, No. 5, 1989, pp. 549-559.

https://doi.org/10.2514/3.10145

[28] Chase, M. W., Curnutt, J. L., Hu, A. T., Prophet, H., Syverud, A. N., and Walker, L. C., "JANAF Thermochemical Tables, 1974 Supplement," Journal of Physical and Chemical Reference Data, Vol. 3, No. 2, 1974, pp. 311-480. https://doi.org/10.1063/1.3253143

[29] Yoshizawa, A., "Statistical Theory for Compressible Turbulent Shear Flows, with the Application to Subgrid Modeling," Physics of Fluids, Vol. 29, No. 7, 1986, pp. 2152-2164. https://doi.org/10.1063/1.865552

[30] Chakravarthy, V., and Menon, S., "Large-Eddy Simulation of Turbulent Premixed Flames in the Flamelet Regime," Combustion Science and Technology, Vol. 162, No. 1, 2001, pp. 175-222. https://doi.org/10.1080/00102200108952141

[31] Fureby, C., Gosman, A., Tabor, G., Weller, H., Sandham, N., and Wolfshtein, M., "Large Eddy Simulation of Turbulent Channel Flows," Turbulent Shear Flows, Vol. 11, Sept. 1997, pp. 28-13.

[32] Li, L., Huang, W., and Yan, L., "Mixing Augmentation Induced by a Vortex Generator Located Upstream of the Transverse Gaseous Jet in Supersonic Flows," Aerospace Science and Technology, Vol. 68, Sept. 2017, pp. 77-89. https://doi.org/10.1016/j.ast.2017.05.016

[33] Huang, W., Liu, W., Li, S., Xia, Z., Liu, J., and Wang, Z., "Influences of the Turbulence Model and the Slot Width on the Transverse Slot Injection Flow Field in Supersonic Flows," Acta Astronautica, Vol. 73, April 2012, pp. 1-9.

https://doi.org/10.1016/j.actaastro.2011.12.003

[34] Weller, H. G., Tabor, G., Jasak, H., and Fureby, C., "A Tensorial Approach to CFD Using Object Oriented Techniques," Computers in Physics, Vol. 12, No. 6, 1998, pp. 620-631. https://doi.org/10.1063/1.168744

[35] Kurganov, A., and Tadmor, E., "New High-Resolution Central Schemes for Nonlinear Conservation Laws and Convection-Diffusion Equations," Journal of Computational Physics, Vol. 160, No. 1, 2000, pp. 241-282. https://doi.org/10.1006/jcph.2000.6459

[36] Baba-Ahmadi, M. H., and Tabor, G., "Inlet Conditions for LES Using Mapping and Feedback Control," Computers and Fluids, Vol. 38, No. 6, 2009, pp. 1299-1311. https://doi.org/10.1016/j.compfluid.2009.02.001

[37] Wu, K., Li, X., Yao, W., and Fan, X., "Three-Dimensional Numerical Study of the Acoustic Properties of a Highly Underexpanded Jet," 20th AIAA International Space Planes and Hypersonic Systems and Technologies Conference, AIAA Paper 2015-3572, 2015.

[38] Li, X., Zhou, R., Yao, W., and Fan, X., "Flow Characteristic of Highly Underexpanded Jets from Various Nozzle Geometries," Applied Thermal Engineering, Vol. 125, Oct. 2017, pp. 240-253. https://doi.org/10.1016/j.applthermaleng.2017.07.002

[39] Li, X., Wu, K., Yao, W., and Fan, X., "A Comparative Study of Highly Underexpanded Nitrogen and Hydrogen Jets Using Large Eddy Simulation," International Journal of Hydrogen Energy, Vol. 41, No. 9, 2016, pp. 5151-5161. https://doi.org/10.1016/j.ijhydene.2016.01.120

[40] Yao, W., Lu, Y., Wu, K., Wang, J., and Fan, X., "Modeling Analysis of an Actively Cooled Scramjet Combustor Under Different Kerosene/Air Ratios," Journal of Propulsion and Power, Vol. 34, No. 4, 2018, pp. $975-991$.

https://doi.org/10.2514/1.B36866

[41] Wu, K., Zhang, P., Yao, W., and Fan, X., "Numerical Investigation on Flame Stabilization in DLR Hydrogen Supersonic Combustor with Strut Injection," Combustion Science and Technology, Vol. 189, No. 12, 2017, pp. 2154-2179. https://doi.org/10.1080/00102202.2017.1365847

[42] Donaldson, C. D., and Snedeker, R. S., "A Study of Free Jet Impingement. Part 1. Mean Properties of Free and Impinging Jets," Journal of Fluid Mechanics, Vol. 45, No. 2, 1971, pp. 281-319. https://doi.org/10.1017/S0022112071000053

[43] Panda, J., and Seasholtz, R. G., "Measurement of Shock Structure and Shock-Vortex Interaction in Underexpanded Jets Using Rayleigh Scattering," Physics of Fluids, Vol. 11, No. 12, 1999, pp. 3761-3777. https://doi.org/10.1063/1.870247

[44] Ashkenas, H., and Sherman, F. S., "Structure and Utilization of Supersonic Free Jets in Low Density Wind Tunnels," NASA CR60423, 1965, https://ntrs.nasa.gov/search.jsp?R=19660017917.

[45] Crist, S., Glass, D. R., and Sherman, P. M., "Study of the Highly Underexpanded Sonic Jet," AIAA Journal, Vol. 4, No. 1, 1966, pp. 68-71. https://doi.org/10.2514/3.3386

[46] Vuorinen, V., Yu, J., Tirunagari, S., Kaario, O., Larmi, M., Duwig, C., and Boersma, B. J., "Large-Eddy Simulation of Highly Underexpanded Transient Gas Jets," Physics of Fluids, Vol. 25, No. 1, 2013, Paper 016101. https://doi.org/10.1063/1.4772192

[47] Krothapalli, A., Buzyna, G., and Lourenco, L., "Streamwise Vortices in an Underexpanded Axisymmetric Jet," Physics of Fluids A: Fluid Dynamics, Vol. 3, No. 8, 1991, pp. 1848-1851. https://doi.org/10.1063/1.857914

[48] Edgington-Mitchell, D., Honnery, D. R., and Soria, J., "The Underexpanded Jet Mach Disk and its Associated Shear Layer," Physics of Fluids, Vol. 26, No. 9, 2014, Paper 096101. https://doi.org/10.1063/1.4894741

[49] Gutmark, E., Schadow, K. C., and Bicker, C. J., "Mode Switching in Supersonic Circular Jets," Physics of Fluids A: Fluid Dynamics, Vol. 1, No. 5,1989 , pp. 868-873. https://doi.org/10.1063/1.857384

[50] Umeda, Y., and Ishii, R., "Existence of Mach Cones and Helical Vortical Structures Around the Underexpanded Circular Jet in the Helical Oscillation Mode," Journal of the Acoustical Society of America, Vol. 112, No. 1, 2002, pp. 99-107. https://doi.org/10.1121/1.1487839

[51] Li, X., He, F., Zhang, X., Hao, P., and Yao, Z., "Shock Motion and Flow Structure of an Underexpanded Jet in the Helical Mode," AIAA Journal, Vol. 57, No. 9, 2019, pp. 3943-3953. https://doi.org/10.2514/1.J058024

[52] Oberleithner, K., Sieber, M., Nayeri, C. N., Paschereit, C. O., Petz, C. Hege, H. C., Noack, B. R., and Wygnanski, I., "Three-Dimensional Coherent Structures in a Swirling Jet Undergoing Vortex Breakdown: Stability Analysis and Empirical Mode Construction," Journal of Fluid Mechanics, Vol. 679, July 2011, pp. 383-414. https://doi.org/10.1017/jfm.2011.141

[53] Ruith, M., Chen, P., Meiburg,E., and Maxworthy, T., "Three-Dimensional Vortex Breakdown in Swirling Jets and Wakes: Direct Numerical Simulation," Journal of Fluid Mechanics, Vol. 486, June 2003, pp. 331-378. https://doi.org/10.1017/S0022112003004749

[54] Berland, J., Bogey, C., and Bailly, C., "Numerical Study of Screech Generation in a Planar Supersonic Jet," Physics of Fluids, Vol. 19, No. 7, 2007, Paper 075105. https://doi.org/10.1063/1.2747225

[55] Raman, G., and Rice, E. J., "Instability Modes Excited by Natural Screech Tones in a Supersonic Rectangular Jet," Physics of Fluids, Vol. 6, No. 12, 1994, pp. 3999-4008. https://doi.org/10.1063/1.868389 